\title{
Average Performance Analysis of Circular and Hyperbolic Geolocation
}

\author{
Andreu Urruela, Student Member, IEEE, Josep Sala, Member, IEEE, and Jaume Riba, Senior Member, IEEE
}

\begin{abstract}
A comparative performance analysis of four geolocation methods in terms of their theoretical root mean square positioning errors is provided. Comparison is established in two different ways: strict and average. In the strict type, methods are examined for a particular geometric configuration of base stations (BSs) with respect to mobile position, which determines a given noise profile affecting the respective time-of-arrival (TOA) or timedifference-of-arrival (TDOA) estimates. In the average type, methods are evaluated in terms of the expected covariance matrix of the position error over an ensemble of random geometries, so that comparison is geometry independent. Exact semianalytical equations and associated lower bounds (depending solely on the noise profile) are obtained for the average covariance matrix of the position error in terms of the so-called information matrix specific to each geolocation method. Statistical channel models inferred from field trials are used to define realistic prior probabilities for the random geometries. A final evaluation provides extensive results relating the expected position error to channel model parameters and the number of base stations.
\end{abstract}

Index Terms-Circular, Cramer-Rao bound (CRB), geolocation, hyperbolic, location, performance analysis, TDOA, TOA.

\section{INTRODUCTION}

T IME-OF-ARRIVAL (TOA) and time-difference-of-arrival (TDOA) measurements are widely used for geolocation applications [1]-[4]. They play a fundamental role in both satellite-based Systems such as Global Positioning System (GPS) GALILEO, and terrestrial-radio-based systems such as Long Range Navigation (LORAN) and other wireless location systems. Location techniques based on TOAs are usually referred to as circular or spherical for 2-D or 3-D location, respectively, while those based on TDOAs are referred to as hyperbolic [5].

Some studies (see [6]-[9] and references therein) have focused on the statistical performance analysis of these location techniques. In general, an analysis of this kind makes a number of assumptions about the nature of the available TOA and TDOA parameters.

Manuscript received April 29, 2003; revised July 19, 2004 and May 10, 2005. This work was financed by the Spanish/Catalan Science and Technology Commissions and FEDER funds from the European Commission under Projects TIC2003-05482 and TEC2004-04526. The review of this paper was coordinated by Dr. R. Klukas.

A. Urruela is with the Signal Processing for Communications Group, Technical University of Catalonia, Barcelona, 08034, Spain (e-mail: andreu.urruela@ ieee.org).

J. Sala is with the Department of Signal Theory and Communications, Technical University of Catalonia, Barcelona, 08034, Spain (e-mail: alvarez@gps. tsc.upc.edu).

J. Riba is with the Department of Signal Theory and Communications and the Signal Processing for Communications Group, Technical University of Catalonia, Barcelona, 08034, Spain (e-mail: jriba@gps.tsc.upc.edu).

Digital Object Identifier 10.1109/TVT.2005.861172
Concerning TOAs, it is important to distinguish between absolute TOAs and pseudo-TOAs. Examples of absolute TOAs are the time-advance (TA) and round-trip-time (RTT) parameters computed in network-based systems. In this case, the circular technique is the only one applicable. The most representative example of the use of pseudo-TOAs is found in satellite-based positioning systems, where they are called pseudoranges. In that case, the receiver clock offset is unknown, and the circular technique is used by including this parameter into the equations.

It is important to consider the procedure used for the computation of TDOAs. One possibility is to take the difference between pairs of pseudo-TOAs, with one particular pseudo-TOA chosen as a reference. A clear example is encountered in the GPS system using the hyperbolic technique [7]. A similar situation is encountered in the uplink TOA method specified for global system for mobile communications (GSM) [8], [10], [11], where a known signal is sent by the mobile and received by several base stations which estimate pseudo-TOAs, with respect to the GPS time. The estimated pseudo-TOAs, along with their timestamps, are relayed back to the mobile location center (MLC), where TDOAs are computed by forming their difference. A more similar case can be found in any code division multiple access (CDMA)-based system as in the observed time difference of arrival (OTDOA) location technique for $3 \mathrm{G}$ mobile communication systems [12], [13] due to the fact that multiple pseudo-TOAs can be measured simultaneously. In all cases, when the TDOAs are obtained in this way, the measurement noises of those sharing a common pseudo-TOA measurement become correlated.

The application of the hyperbolic technique to pseudo-TOA measurements has been analyzed in the literature. When the receiver clock offset is modeled as an additional deterministic unknown parameter, it has been demonstrated in [7] that both approaches (circular and hyperbelic on TDOA measurements) yield identical estimates, although their authors considered only the special case in which all TOA estimates are uncorrelated and have equal variance. On the other hand, it is demonstrated in [9] that the Cramér-Rao (CRB) bounds for position accuracy using TDOA measurements approach those of the spherical navigation system as the variance in the clock-offset approaches zero and those of the hyperbolic positioning system as the variance of the clock offset approaches infinity. Note that clock-offset variance includes both the inacuracies of the local clock, as well as the incuracies in the common transmit time of the BSs.

There exists in practice an alternative to the computation of TDOAs. In the downlink enhanced observed time difference (E-OTD) [13] scheme, for instance, timing differences are estimated directly at the mobile station, one at a time, as only 
two RF chains are implemented in mobile terminals. In this case, different TDOAs are generated at different times, leading to higher latency and uncorrelated estimates if the estimation period is longer than the typical coherence time of the mobile radio channel [8]. It is noted, in that case, that small mobile movements (on the order of the wavelength) during two consecutive TDOA measurements suffice to decorrelate them. However, in the analysis of this alternative, one can still assume that the mobile station is essentially stationary during the complete estimation period, which will be $N-1$ times higher than a single TDOA measurement time, where $N$ is the number of beacons. This assumption is implied by the fact that the maximum change of position of the mobile within the complete estimation period is not significant in comparison with the expected positioning accuracy in the multipath environment. It is finally noted that, when using only two RF chains, the direct computation of TDOAs is more practical than that of pseudo-TOAs, because the computation of pseudo-TOAs at different times requires time stamping due to the receiver clock drift, which is not necessary for the computation of TDOAs.

Therefore, in the performance analysis of the hyperbolic technique, the modeling of TDOA errors may depend on the application, which has a clear influence on their cross-correlation structure. The first contribution of this paper is the complete performance comparison between the circular and hyperbolic techniques for all aforementioned cases. The second contribution is the analysis of the average performance (and lower bound) over all the geometric configurations of the base stations. To our knowledge, this average analysis has never been reported, and it proves very useful for comparing the expected accuracy of different location techniques, with independence of the particular geometric configuration of the base stations. This analysis is limited to the case where the beacons are uniformly distributed over the plane, although it can be generalized to three dimensional distributions.

\section{DEFINITION OF GEOLOCATION SCENARIOS}

In this paper, the accuracy of each of the four geolocation methods considered is defined in terms of their position error covariance matrices

$$
\begin{aligned}
& \mathbf{C}_{\mathrm{st}} \text { : Circular with known clock } \\
& \mathbf{C}_{\mathrm{s} \overline{\mathrm{t}}}: \text { Circular with unknown clock } \\
& \mathbf{C}_{\mathrm{hc}}: \text { Hyperbolic correlated } \\
& \mathbf{C}_{\mathrm{h} \overline{\mathrm{c}}}: \text { Hyperbolic uncorrelated. }
\end{aligned}
$$

1) Circular technique with known clock offset: In this scenario, the available measurements consist of the absolute TOAs of the signals transmitted between the mobile and the beacons. A circular technique is used to compute the position estimate. The covariance matrix of the position error is denoted $\mathbf{C}_{\text {st }}$.

2) Circular technique with unknown clock offset: In this scenario, the available measurements consist of the pseudoTOAs of the signals transmitted between the mobile and the beacons. A circular technique is used to compute the position estimate along with the clock offset. The covariance matrix of the position error is denoted $\mathbf{C}_{\mathrm{s} \overline{\mathrm{t}}}$.

3) Hyperbolic technique with correlated noise: In this scenario, the available measurements consist of the TDOAs obtained from differences between pairs of pseudo-TOAs. A hyperbolic technique is used to compute the position estimate. The covariance matrix of the position error is denoted $\mathbf{C}_{\mathrm{hc}}$.

4) Hyperbolic technique with uncorrelated noise: In this scenario, the available measurements consist of uncorrelated TDOAs obtained at different times. A hyperbolic technique is used to compute the position estimate. The covariance matrix of the position error is denoted $\mathbf{C}_{\mathrm{h} \bar{c}}$. Note that this is the case of downlink E-OTD, as described in Section I, where TDOA measurements are computed one at a time to avoid the impact of clock drift.

Finally, note that in scenarios with hardware constraints (frequency division multiple access (FDMA) systems with only two RF chains, as in GSM), measurements cannot be taken simultaneously. In this case, the uncorrelated hyperbolic approach appears in a natural way, where TDOA measurements are computed one at a time. In these scenarios (two RF chains), pseudo-TOAs and TOAs can also be measured sequentially, but clock drift affects performance.

\section{REVIEW AND EXTENSION OF PERFORMANCE LIMITS}

\section{A. Signal Model for Circular Methods}

In the general case, the TOA/pseudo-TOA measurements obtained from $N$ beacons can be expressed as a function of the mobile position $\mathbf{x}=\left[x_{1}, x_{2}\right]^{T}$ as follows:

$$
\begin{aligned}
\hat{t}_{n} & =\tilde{t}_{n}\left(\mathbf{x}, t_{o}\right)+u_{n} \\
& =t_{n}(\mathbf{x})+t_{o}+u_{n}
\end{aligned}
$$

where $1 \leq n \leq N, \tilde{t}_{n}\left(\mathbf{x}, t_{o}\right)$ is defined in the trivial way, $t_{n}(\mathbf{x})=\frac{1}{c}\left\|\mathbf{x}-\mathbf{x}_{n}\right\|, \mathbf{x}_{n}=\left[x_{1, n}, x_{2, n}\right]^{T}$ is the $n$th beacon position, $t_{o}$ is the clock offset, which has the same value for all $n, u_{n}$ are the measurement errors, and $c$ is the speed of light. Note that $t_{o}=0$ in the case of absolute TOAs, while it becomes an unknown parameter in the case of pseudo-TOAs. Note also that the effect of clock drift of the mobile is inherently not considered in (1) and (2). This is, in general, an unfair comparison, since as has been said, the hyperbolic uncorrelated approach is the single robust approach in terms of clock drift under hardware constraints. It is out of the scope of this paper to show the effect of such phenomena.

If the time interval used to perform these measurements is sufficiently large (see [14] and references therein), then, by the central limit theorem, we can approximate the terms $u_{n}$ as jointly Gaussian. Further, as the present contribution is focused only on the computation of performance limits, it will be assumed that the possible bias of the measurements caused by hardware calibration errors and multipath effects is known, which is equivalent to assuming (as in [8]) that the measurement errors $u_{n}$ are zero-mean. An additional rationale for this assumption is based 
on the fact that we are interested on an average performance analysis. Therefore, by considering the ensemble of all possible random biases affecting all measurements $\hat{t}_{n}$, there always exists an equivalent variance amplification (of the noise term $u_{n}$ ) producing, on average, an equivalent global effect. This feature is very appealing, as it allows modeling all error sources simply as an additional zero mean term with a specified variance. A similar rationale applies to hardware calibration. For an analysis of the impact of biased measurements on the accuracy of position location methods, see [15] and [14].

Let the measurements be stacked into vector $\hat{\mathbf{t}}$. Then, from (1) and (2)

$$
\begin{aligned}
\hat{\mathbf{t}} & =\tilde{\mathbf{t}}\left(\mathbf{x}, t_{o}\right)+\mathbf{u} \\
& =\mathbf{t}(\mathbf{x})+t_{o} \mathbf{1}+\mathbf{u}
\end{aligned}
$$

where 1 is the all-ones column vector, and the remaining vectors become defined in a trivial way from (2). The $N \times N$ covariance matrix of the noise vector $\mathbf{u}$ is

$$
\mathbf{R}=E\left[\mathbf{u u}^{T}\right] .
$$

It is assumed throughout the paper that matrix $\mathbf{R}$ is known, which allows the establishment of lower performance bounds. The statistical distribution of the (diagonal) matrix $\mathbf{R}$ has been obtained from models based on real data [16], and is detailed in Section VII-B. In practice, $\mathbf{R}$ is to be estimated, with the consequent degradation in the performance predicted by the bounds derived herein. Nonetheless, they are still valid as lower bounds.

\section{B. Signal Model for Hyperbolic Methods}

The $N-1$ TDOA measurements are given by

$$
\begin{aligned}
\hat{\mathbf{d}} & =\mathbf{H} \tilde{\mathbf{t}}\left(\mathbf{x}, t_{o}\right)+\mathbf{v} \\
& =\mathbf{H t}(\mathbf{x})+\mathbf{v}
\end{aligned}
$$

with $\mathbf{H}$ repersenting any full-rank $(N-1) \times N$ matrix such that $\mathbf{H 1}=\mathbf{0}$, where $\mathbf{0}$ is the all-zeros column vector. Note that this condition implies that the measurement vector $\hat{\mathbf{d}}$ is not affected by the presence of the nonzero clock offset $t_{o}$ in (7). The most common example of $\mathbf{H}$ is

$$
\mathbf{H}=[\mathbf{1},-\mathbf{I}]
$$

where all TDOAs are obtained with respect to a common pseudo-TOA. This first (or reference) pseudo-TOA corresponds to the all-ones column of $\mathbf{H}$. will henceforth be called the pivot BS. Nonetheless, the analysis in this paper is general for any $\mathbf{H}$, unless otherwise stated. The $(N-1) \times(N-1)$ covariance matrix of the noise vector $\mathbf{v}$ is

$$
\mathbf{R}_{\mathrm{h}}=E\left[\mathbf{v v}^{\mathbf{T}}\right] .
$$

Note that, only in the "hyperbolic-correlated" scenario, the TDOAs are computed as $\hat{\mathbf{d}}=\mathbf{H} \hat{\mathbf{t}}$, and then it holds from (4) that $\mathbf{v}=\mathbf{H u}$, and

$$
\mathbf{R}_{\mathrm{h}}=\mathbf{R}_{\mathrm{hc}}=\mathbf{H R H}^{T} .
$$

However, in the "hyperbolic-uncorrelated" scenario, the structures of $\mathbf{R}_{\mathrm{h}}=\mathbf{R}_{\mathrm{h} \bar{c}}$ and $\mathbf{R}$ are not necessarily related in a simple way. Indeed, in the "hyperbolic-uncorrelated" scenario, matrix $\mathbf{H}$ in (7) only shows how the pairs of BS are selected to obtain the $N-1$ TDOA measurements, but measurements remain independent. In that scenario, the relationship between $\mathbf{R}_{\mathrm{h} \bar{c}}$ and $\mathbf{R}$ for any $\mathbf{H}$ can be expressed as

$$
\mathbf{R}_{\mathrm{h}}=\mathbf{R}_{\mathrm{h} \overline{\mathrm{c}}}=\left(\mathbf{H R H}^{T}\right) \odot \mathbf{I}
$$

where $\odot$ stands for the component-wise Schur-Hadamard product between two matrices. Note that $\mathbf{R}_{\mathrm{hc}}$ and $\mathbf{R}_{\mathrm{h} \bar{c}}$ are the specific versions of $\mathbf{R}_{\mathrm{h}}$ for the correlated and uncorrelated version, respectively, of the hyperbolic approach.

\section{Performance Limits}

The performance limits are computed as the performance of the linearized weighted least squares (WLS) estimator, which coincides with the Cramer-Rao bound in the case of Gaussian noise.

For circular approaches, the linearization of (4) at the true position yields

$$
\Delta \hat{\mathbf{t}}=\mathbf{F} \Delta \mathbf{x}+\Delta t_{o} \mathbf{1}+\mathbf{u}
$$

where the $N \times 2$ matrix of partial derivatives $\mathbf{F}$ can be calculated as

$$
\begin{aligned}
{[\mathbf{F}]_{n, i} } & =\frac{\partial t_{n}(\mathbf{x})}{\partial x_{i}}=\frac{1}{c} \frac{x_{i}-x_{i, n}}{\left\|\mathbf{x}-\mathbf{x}_{n}\right\|} \\
& =\frac{1}{c} \frac{x_{i}-x_{i, n}}{\sqrt{\left(x_{1}-x_{1, n}\right)^{2}+\left(x_{2}-x_{2, n}\right)^{2}}}
\end{aligned}
$$

with $i=1,2$, and where $[\mathbf{M}]_{l, l^{\prime}}$ denotes the $l$ th row $l^{\prime}$ th column element of a matrix $\mathrm{M}$. The WLS estimator of $\Delta \mathrm{x}$ and $\Delta t_{o}$ is the minimizer of

$$
\left(\Delta \hat{\mathbf{t}}-\mathbf{F} \Delta \mathbf{x}-\Delta t_{o} \mathbf{1}\right)^{T} \mathbf{R}^{-1}\left(\Delta \hat{\mathbf{t}}-\mathbf{F} \Delta \mathbf{x}-\Delta t_{o} \mathbf{1}\right)
$$

which, using standard differential matrix calculus, can be expressed as

$$
\left[\begin{array}{c}
\Delta \mathbf{x} \\
\Delta t_{o}
\end{array}\right]=\left(\mathbf{F}_{1}^{T} \mathbf{R}^{-1} \mathbf{F}_{1}\right)^{-1} \mathbf{F}_{1}^{T} \mathbf{R}^{-1} \boldsymbol{\Delta} \hat{\mathbf{t}}
$$

where $\mathbf{F}_{1}=[\mathbf{F}, \mathbf{1}]$, and the covariance matrix of $\Delta \mathbf{x}$ is given by

$$
\mathbf{C}_{\mathrm{s} \overline{\mathrm{t}}}=\mathbf{T}^{T}\left(\mathbf{F}_{1}^{T} \mathbf{R}^{-1} \mathbf{F}_{1}\right)^{-1} \mathbf{T}
$$

with $\mathbf{T}^{T}=[\mathbf{I}, \mathbf{0}]$. Following the same procedure, the covariance matrix of $\Delta \mathrm{x}$ in the case of known clock $t_{o}$ is

$$
\mathbf{C}_{\mathrm{st}}=\left(\mathbf{F}^{T} \mathbf{R}^{-1} \mathbf{F}\right)^{-1} \text {. }
$$

On the other hand, for hyperbolic approaches, we have the linearization of (7) that can be written as

$$
\Delta \hat{\mathbf{d}}=\mathbf{H F} \Delta \mathbf{x}+\mathbf{v}
$$

and the WLS estimator of $\Delta \mathrm{x}$ becomes the minimizer of

$$
(\Delta \hat{\mathbf{d}}-\mathbf{H F} \Delta \mathbf{x})^{T} \mathbf{R}_{\mathrm{h}}^{-1}(\Delta \hat{\mathbf{d}}-\mathbf{H F} \Delta \mathbf{x})
$$

given by

$$
\Delta \mathbf{x}=\left(\mathbf{F}^{T} \mathbf{H}^{T} \mathbf{R}_{\mathrm{h}}^{-1} \mathbf{H F}\right)^{-1} \mathbf{F}^{T} \mathbf{H}^{T} \mathbf{R}_{\mathrm{h}}^{-1} \Delta \hat{\mathbf{d}}
$$


with covariance matrix

$$
\mathbf{C}_{\mathrm{h}}=\left(\mathbf{F}^{T} \mathbf{H}^{T} \mathbf{R}_{\mathrm{h}}^{-1} \mathbf{H F}\right)^{-1}
$$

where the subindex in $\mathbf{C}_{\mathrm{h}}$ and $\mathbf{R}_{\mathrm{h}}$ stands for a generic hyperbolic approach. For the uncorrelated hyperbolic approach (11), this covariance matrix can be expressed as

$$
\begin{aligned}
& \mathbf{C}_{\mathrm{h} \overline{\mathrm{c}}}=\left(\mathbf{F}^{T} \mathbf{H}^{T} \mathbf{R}_{\mathrm{h} \overline{\mathrm{c}}}^{-1} \mathbf{H F}\right)^{-1} \\
& \mathbf{C}_{\mathrm{h} \overline{\mathrm{c}}}=\left(\mathbf{F}^{T} \mathbf{H}^{T}\left[\left(\mathbf{H R H} \mathbf{H}^{T}\right) \odot \mathbf{I}\right]^{-1} \mathbf{H F}\right)^{-1}
\end{aligned}
$$

and, in the specific case that the TDOAs are computed as differences between pseudo-TOAs (correlated hyperbolic approach), it holds from (10) that $\mathbf{R}_{\mathrm{h}}=\mathbf{R}_{\mathrm{hc}}=\mathbf{H} \mathbf{R} \mathbf{H}^{T}$, and the corresponding covariance matrix is given by

$$
\begin{aligned}
& \mathbf{C}_{\mathrm{hc}}=\left(\mathbf{F}^{T} \mathbf{H}^{T} \mathbf{R}_{\mathrm{hc}}^{-1} \mathbf{H F}\right)^{-1} \\
& \mathbf{C}_{\mathrm{hc}}=\left(\mathbf{F}^{T} \mathbf{H}^{T}\left(\mathbf{H} \mathbf{R} \mathbf{H}^{T}\right)^{-1} \mathbf{H F}\right)^{-1} .
\end{aligned}
$$

\section{COMPARISON CRITERIA}

Strict and average comparison are carried out in this paper. Such comparison between two methods can be established in terms of the positive definite character of the respective covariance matrices, or in a less restrictive sense, in terms of their trace. By strict, we mean a comparison between two geolocation methods for a specific geometry of the base stations with respect to the mobile station, and for a specific noise profile affecting either TOA or TDOA measurements. By average, we mean a comparison between the expected covariance matrices over a joint ensemble of geometries and noise profiles. Thus, a geolocation method $A$ is said to be strictly better than (or superior to) a geolocation method $B$ when the respective covariance matrices fulfil $\mathbf{C}_{A} \leq \mathbf{C}_{B}$ (i.e., $\mathbf{C}_{B}-\mathbf{C}_{A}$ is nonnegative definite) for any geometry or noise profile. If two geolocation methods $A$ and $B$ cannot be guaranteed to fulfill the strict superiority condition, comparison must be established in terms of their average behavior, i.e., $E_{\mathcal{G}} \mathbf{C}_{A} \leq E_{\mathcal{G}} \mathbf{C}_{B}$, in terms of the expectation over the geometry and noise profile ensemble $(\mathcal{G})$. Then, "strictly" is dropped, and we state that the geolocation method $A$ is better than the geolocation method $B$. Hence, strict superiority implies (average) superiority, but not vice-versa. The choice of implementing a geolocation method $A$ over a method $B$ must necessarily be governed by an average superiority criterion, which will guarantee a global improvement for a number of scenarios, although for some specific geometries, $B$ may outperform $A$. Average performance analysis will require (Section VI) the definition of a reasonable probability density function for the random geometries and noise profiles.

\section{Strict COMPARISON OF LOCATION TECHNIQUES}

This section establishes the relationship among the four geolocation methods previously described in terms of strict superiority. We establish the following results:
A) $\mathbf{C}_{\mathrm{s} \overline{\mathrm{t}}}=\mathbf{C}_{\mathrm{hc}}$;
B) $\mathbf{C}_{\mathrm{s} \overline{\mathrm{t}}} \geq \mathbf{C}_{\mathrm{st}}$;

C) the relationship between $\mathbf{C}_{\mathrm{hc}}$ and $\mathbf{C}_{\mathrm{h} \overline{\mathrm{c}}}$ depends on the specific geometry and noise profile. Comparison must be established in terms of the respective averages (Section VI).

\section{A. Equality Between $\mathbf{C}_{\mathrm{s} \overline{\mathrm{t}}}$ and $\mathbf{C}_{\mathrm{hc}}$}

According to (17) and (26), $\mathbf{C}_{\mathrm{s} \overline{\mathrm{t}}}^{-1}$ and $\mathbf{C}_{\mathrm{hc}}^{-1}$ can be expressed as

$$
\begin{aligned}
& \mathbf{C}_{\mathrm{s} \overline{\mathrm{t}}}^{-1}=\left(\mathbf{T}^{T}\left(\mathbf{F}_{1}^{T} \mathbf{R}^{-1} \mathbf{F}_{1}\right)^{-1} \mathbf{T}\right)^{-1} \\
& \mathbf{C}_{\mathrm{hc}}^{-1}=\mathbf{F}^{\mathbf{T}}\left(\mathbf{H}^{\mathbf{T}}\left(\mathbf{H} \mathbf{R} \mathbf{H}^{T}\right)^{-1} \mathbf{H}\right) \mathbf{F}^{\mathbf{T}} .
\end{aligned}
$$

Several manipulations will show that they are indeed equal. We start forming the product $\mathbf{F}_{1}^{T} \mathbf{R}^{-1} \mathbf{F}_{1}$ in $\mathbf{C}_{\mathrm{s} \overline{\mathrm{t}}}^{-1}$, then

$$
\begin{aligned}
\mathbf{F}_{1}^{T} \mathbf{R}^{-1} \mathbf{F}_{1} & =\left[\begin{array}{c}
\mathbf{F}^{T} \\
\mathbf{1}^{T}
\end{array}\right] \mathbf{R}^{-1}\left[\begin{array}{ll}
\mathbf{F}
\end{array}\right] \\
& =\left[\begin{array}{ll}
\mathbf{F}^{T} \mathbf{R}^{-1} \mathbf{F} & \mathbf{F}^{T} \mathbf{R}^{-1} \mathbf{1} \\
\mathbf{1}^{T} \mathbf{R}^{-1} \mathbf{F} & \mathbf{1}^{T} \mathbf{R}^{-1} \mathbf{1}
\end{array}\right]
\end{aligned}
$$

Using block matrix inversion [17], [18] in (29)

$$
\begin{aligned}
\left(\mathbf{F}_{1}^{T} \mathbf{R}^{-1} \mathbf{F}_{1}\right)^{-1} & =\left[\begin{array}{cc}
\mathbf{B}_{11} & \mathbf{b}_{12} \\
\mathbf{b}_{21}^{H} & \beta
\end{array}\right] \\
\mathbf{B}_{11} & =\left[\mathbf{F}^{T} \mathbf{R}^{-1} \mathbf{F}-\frac{\mathbf{F}^{T} \mathbf{R}^{-1} \mathbf{1} \mathbf{1}^{T} \mathbf{R}^{-1} \mathbf{F}}{\mathbf{1}^{T} \mathbf{R}^{-1} \mathbf{1}}\right]^{-1}
\end{aligned}
$$

but we also have from (27) that

$$
\begin{aligned}
\mathbf{C}_{\mathrm{s} \overline{\mathrm{t}}}^{-1} & =\left[\left[\begin{array}{ll}
\mathbf{I} & \mathbf{0}
\end{array}\right]\left(\mathbf{F}_{1}^{T} \mathbf{R}^{-1} \mathbf{F}_{1}\right)^{-1}\left[\begin{array}{c}
\mathbf{I} \\
\mathbf{0}^{T}
\end{array}\right]\right]^{-1}=\mathbf{B}_{11}^{-1} \\
\mathbf{B}_{11}^{-1} & =\mathbf{F}^{T} \mathbf{R}^{-1} \mathbf{F}-\frac{\mathbf{F}^{T} \mathbf{R}^{-1} \mathbf{1} \mathbf{1}^{T} \mathbf{R}^{-1} \mathbf{F}}{\mathbf{1}^{T} \mathbf{R}^{-1} \mathbf{1}} \\
& =\mathbf{F}^{T}\left(\mathbf{R}^{-1}-\frac{\mathbf{R}^{-1} \mathbf{1 1}^{T} \mathbf{R}^{-1}}{\mathbf{1}^{T} \mathbf{R}^{-1} \mathbf{1}}\right) \mathbf{F}
\end{aligned}
$$

Therefore, comparing (28) and (30), and using the fact that

$$
\mathbf{R}^{-1}-\frac{\mathbf{R}^{-1} \mathbf{1 1}^{T} \mathbf{R}^{-1}}{\mathbf{1}^{T} \mathbf{R}^{-1} \mathbf{1}}=\mathbf{H}^{T}\left(\mathbf{H} \mathbf{R} \mathbf{H}^{T}\right)^{-1} \mathbf{H}
$$

is true for any matrix $\mathbf{H}$ that fulfills $\mathbf{H 1}=\mathbf{0}$ (see Appendix F), we have that

$$
\mathbf{C}_{\mathrm{s} \overline{\mathrm{t}}}=\mathbf{C}_{\mathrm{hc}}
$$

which generalizes the work presented in [7], in the sense that (32) is valid for any $\mathbf{R}$.

\section{B. Strict Superiority Between $\mathbf{C}_{\mathrm{s} \overline{\mathrm{t}}}$ and $\mathbf{C}_{\mathrm{st}}$}

We want to compare the matrices defined in (17) and (18)

$$
\begin{aligned}
& \mathbf{C}_{\mathrm{s} \overline{\mathrm{t}}}=\mathbf{T}^{T}\left(\mathbf{F}_{1}^{T} \mathbf{R}^{-1} \mathbf{F}_{1}\right)^{-1} \mathbf{T} \\
& \mathbf{C}_{\mathrm{st}}=\left(\mathbf{F}^{T} \mathbf{R}^{-1} \mathbf{F}\right)^{-1}
\end{aligned}
$$

to prove that $\mathbf{C}_{\mathrm{s} \overline{\mathrm{t}}}>\mathbf{C}_{\mathrm{st}}$ for any geometry or noise profile. We know from (30) that

$$
\mathbf{C}_{\mathrm{s} \overline{\mathrm{t}}}^{-1}=\mathbf{F}^{T}\left(\mathbf{R}^{-1}-\frac{\mathbf{R}^{-1} \mathbf{1} \mathbf{1}^{T} \mathbf{R}^{-1}}{\mathbf{1}^{T} \mathbf{R}^{-1} \mathbf{1}}\right) \mathbf{F} .
$$


Hence

$$
\mathbf{C}_{\mathrm{s} \overline{\mathrm{t}}}^{-1}=\mathbf{C}_{\mathrm{st}}^{-1}-\mathbf{q q}^{T}
$$

where

$$
\mathbf{q}=\frac{\mathbf{F}^{T} \mathbf{R}^{-1} \mathbf{1}}{\sqrt{\mathbf{1}^{T} \mathbf{R}^{-1} \mathbf{1}}}
$$

Using the matrix inversion lemma in (34), we see that $\mathbf{C}_{\mathrm{s} \overline{\mathrm{t}}}$ is more positive definite than $\mathbf{C}_{\mathrm{st}}$ (i.e., $\mathbf{C}_{\mathrm{s} \overline{\mathrm{t}}}-\mathbf{C}_{\mathrm{st}}$ is a positive definite matrix),

$$
\mathbf{C}_{\mathrm{s} \overline{\mathrm{t}}}=\mathbf{C}_{\mathrm{st}}+\frac{\mathbf{q} \mathbf{q}^{T}}{1+\mathbf{q}^{T} \mathbf{C}_{\mathrm{st}} \mathbf{q}} \geq \mathbf{C}_{\mathrm{st}}
$$

and we conclude from (36) that absolute TOA measurements generate a $\mathbf{C}_{\mathrm{st}}$ strictly contained in $\mathbf{C}_{\mathrm{s} \bar{t}}$ for any geometry or noise correlation. Note, however, that $\mathbf{C}_{\mathrm{st}}$ and $\mathbf{C}_{\mathrm{s} \overline{\mathrm{t}}}$ are not simply related by a scaling factor.

\section{Relationship Between $\mathbf{C}_{\mathrm{hc}}$ and $\mathbf{C}_{\mathrm{h} \overline{\mathrm{c}}}$}

We show in this section that strict comparison is not possible between $\mathbf{C}_{\mathrm{hc}}$ and $\mathbf{C}_{\mathrm{h} \bar{c}}$, as some scenarios will deliver better performance for $\mathbf{C}_{\mathrm{hc}}$ and others will not. The judgement as to what method is better, and to what extent, will require the average performance analysis in Section VI for an ensemble of geometries and noise profiles. The derivation here is rather involved, and most of it is developed in the appendices (see Appendices $\mathrm{A}$ and $\mathrm{B}$. The relationship between $\mathbf{C}_{\mathrm{hc}}$ and $\mathbf{C}_{\mathrm{h} \overline{\mathrm{c}}}$ depends on the way in which the TDOAs are obtained, as this determines the respective noise profile. Hence, the measurement noise correlation matrices will be defined first. The TOA noise correlation matrix $\mathbf{R}$ is defined by

$$
\begin{aligned}
\mathbf{R} & =\operatorname{diag}\left(h_{1}^{2}, h_{2}^{2}, \ldots, h_{N}^{2}\right)=\sigma^{2} \mathbf{D}_{c} \\
\sigma^{2} & =\sum_{i=1}^{N} h_{i}^{2} \\
\mathbf{D}_{c} & =\operatorname{diag}\left(s_{1}^{2}, s_{2}^{2}, \ldots, s_{N}^{2}\right) \\
s_{i}^{2} & =h_{i}^{2} / \sigma^{2}
\end{aligned}
$$

where $\sigma^{2}$ constitutes the global noise power and $s_{i}^{2}$ the components of the noise profile, with $\sum_{i=1}^{N} s_{i}^{2}=1$. The uncorrelated hyperbolic correlation matrix $\mathbf{R}_{\mathrm{h} \bar{c}}$ associated with the noise profile of $\mathbf{R}$ and defined in (11) depends on how pseudo-TOA differences are taken. Indeed, this is the role of matrix $\mathbf{H}$ in (11). For differences taken using $\mathbf{H}=[\mathbf{1},-\mathbf{I}]$, which is the most common case in real implementations, we set $\mathbf{R}_{\mathrm{h} \overline{\mathrm{c}}}$ from (11) as

$$
\begin{aligned}
\mathbf{R}_{\mathrm{h} \overline{\mathrm{c}}} & =\operatorname{diag}\left(h_{\bar{c}, 1}^{2}, h_{\bar{c}, 2}^{2}, \ldots, h_{\bar{c}, N-1}^{2}\right)=\sigma_{\mathrm{h} \overline{\mathrm{c}}}^{2} \mathbf{D}_{\overline{\mathrm{c}}} \\
\sigma_{\mathrm{h} \overline{\mathrm{c}}}^{2} & =\sum_{i=1}^{N-1} h_{\bar{c}, i}^{2} \\
h_{\bar{c}, i}^{2} & =h_{1}^{2}+h_{i+1}^{2} \\
\mathbf{D}_{\overline{\mathrm{c}}} & =\operatorname{diag}\left(s_{\bar{c}, 1}^{2}, s_{\bar{c}, 2}^{2}, \ldots, s_{\bar{c}, N-1}^{2}\right) \\
s_{\bar{c}, i}^{2} & =h_{\bar{c}, i}^{2} / \sigma_{\mathrm{h} \overline{\mathrm{c}}}^{2}=\frac{\sigma^{2}}{\sigma_{\mathrm{h} \overline{\mathrm{c}}}^{2}}\left(s_{1}^{2}+s_{i+1}^{2}\right)
\end{aligned}
$$

where the noise for each TDOA is the addition of the respective pseudo-TOA noise components. It is assumed here that each pair of pseudo-TOA measurements used to determine a single TDOA are not performed simultaneously, as would be the case for $\mathbf{R}_{\text {hc }}$. Instead, each pair of TDOA measurements are carried out in different time intervals, such that the offdiagonal terms of $\mathbf{R}_{\mathrm{h} \overline{\mathrm{c}}}$ are necessarily zero for lack of correlation, as shown in (11). Hence, generally $\sigma_{\mathrm{h} \bar{c}}^{2}$ is on the order of $2 \sigma^{2}$. This will set an absolute criterion for comparing both methods. Now, it is shown in Appendix A that $\mathbf{C}_{\mathrm{hc}}$ and $\mathbf{C}_{\mathrm{h} \bar{c}}$ can be expressed as

$$
\begin{aligned}
& \mathbf{C}_{\mathrm{hc}}^{-1}=\mathbf{F}_{\mathrm{u}}^{T} \mathbf{I}_{N-1} \mathbf{F}_{\mathrm{u}} \\
& \mathbf{C}_{\mathrm{h} \overline{\mathrm{c}}}^{-1}=\mathbf{F}_{\mathrm{u}}^{T}\left(\frac{\sigma^{2}}{\sigma_{\mathrm{h} \overline{\mathrm{c}}}^{2}} \boldsymbol{\Lambda}\right) \mathbf{F}_{\mathrm{u}}
\end{aligned}
$$

where

$$
\frac{\sigma^{2}}{\sigma_{\mathrm{h} \overline{\mathrm{c}}}^{2}} \operatorname{tr}\{\boldsymbol{\Lambda}\}=\operatorname{tr}\left\{\mathbf{I}_{N-1}\right\}=N-1
$$

and where $\mathbf{I}_{N-1}$ is the $N-1 \times N-1$ identity matrix, and $\mathbf{F}_{\mathrm{u}}$ is a geometry-dependent matrix obtained from $\mathbf{F}$ (see Appendix A) as $\mathbf{F}_{\mathrm{u}}=\mathbf{W}^{T} \mathbf{F}$, with $\mathbf{W}$ a geometry-independent matrix of rank $N-1$. $\left(\sigma^{2} / \sigma_{\mathrm{h} \bar{c}}^{2}\right) \boldsymbol{\Lambda}$ is a positive definite diagonal matrix with $N-1$ nonzero eigenvalues, such that some are larger than 1 and some smaller than 1 to fulfill the trace constraint (43). We can see from this that according to each specific geometry and noise profile, depending on how $\mathbf{F}$ is projected onto the subspace spanned by the vectors of $\mathbf{W}$, the Euclidean norm of each column in $\mathbf{F}_{\mathrm{u}}$ will be affected in a different way in (41) and (42). So, nothing in terms of the strict superiority between the matrices $\mathbf{C}_{\mathrm{hc}}$ and $\mathbf{C}_{\mathrm{h} \bar{c}}$ can be stated.

An alternative way to show that $\mathbf{C}_{\mathrm{hc}}$ is not stricly superior to $\mathbf{C}_{\mathrm{h} \overline{\mathrm{c}}}$ or vice versa is by checking that $\mathbf{C}_{\mathrm{hc}}-\mathbf{C}_{\mathrm{h} \overline{\mathrm{c}}}$ is neither positive nor negative definite (i.e., it has both positive and negative eigenvalues). This can be easily checked in terms of the difference between their inverses because if $\mathbf{C}_{\mathrm{hc}}^{-1}-\mathbf{C}_{\mathrm{h} \overline{\mathrm{c}}}^{-1}$ has both positive and negative eigenvalues, then, so does $\mathbf{C}_{\mathrm{hc}}-\mathbf{C}_{\mathrm{h} \bar{c}}$. ${ }^{1}$

$$
\begin{aligned}
\mathbf{C}_{\mathrm{hc}}^{-1}-\mathbf{C}_{\mathrm{h} \overline{\mathrm{c}}}^{-1} & =\mathbf{F}_{\mathrm{u}}^{T}\left[\mathbf{I}_{N-1}-\left(\frac{\sigma^{2}}{\sigma_{\mathrm{h} \bar{c}}^{2}} \boldsymbol{\Lambda}\right)\right] \mathbf{F}_{\mathrm{u}} \\
& =\mathbf{F}_{\mathrm{u}}^{T} \Lambda^{\prime} \mathbf{F}_{\mathrm{u}} .
\end{aligned}
$$

Since from the constraint in (43) $\left(\left(\sigma^{2} / \sigma_{\mathrm{h} \bar{c}}^{2}\right) \boldsymbol{\Lambda}\right)$ is a diagonal matrix with positive elements larger and smaller than one, $\Lambda^{\prime}$ must be a diagonal matrix with positive and negative elements. Hence, it is proved that $\mathbf{C}_{\mathrm{hc}}^{-1}-\mathbf{C}_{\mathrm{h} \bar{c}}^{-1}$ and, consequently, $\mathbf{C}_{\mathrm{hc}}-\mathbf{C}_{\mathrm{h} \overline{\mathrm{c}}}$, have positive and negative eigenvalues (they are neither positive nor negative definite). This can be mathematically expressed as

$$
\begin{aligned}
& \mathbf{C}_{\mathrm{hc}} \ngtr \mathbf{C}_{\mathrm{h} \overline{\mathrm{c}}} \\
& \mathbf{C}_{\mathrm{hc}} \nless \mathbf{C}_{\mathrm{h} \overline{\mathrm{c}}} .
\end{aligned}
$$

\footnotetext{
${ }^{1}$ This results from the following property regarding two positive definite matrices $\mathbf{A}_{1}$ and $\mathbf{A}_{2}: \mathbf{A}_{1} \geq \mathbf{A}_{2}$ if and only if $\mathbf{A}_{1}^{-1} \leq \mathbf{A}_{2}^{-1}$. Therefore, if $\mathbf{A}_{1}-\mathbf{A}_{2}$ has positive and negative eigenvalues, $\mathbf{A}_{1}^{-1}-\mathbf{A}_{2}^{-1}$ must as well. Otherwise, a contradiction with the previous property would occur.
} 


\section{Average COMPARISON OF LocATion TECHNiQues}

Strict performance comparison of circular and hyperbolic geolocation in terms of the covariance matrix of the position error has been developed in Section V. It has also been shown that at least one case exists (uncorrelated hyperbolic geolocation) where two methods do not compare in the strict sense, due to their dependence on each particular geometry. In this and other cases, comparison may be established only in terms of the expected performance over a random geometry.

This section presents an efficient procedure for comparing algorithms in terms of their average covariance matrix, as well as an analycal lower bound in the positive definite sense. The placement of BSs is defined according to a uniform random distribution on a circular area centered at the true mobile position, and depending on a density parameter $\rho\left(\mathrm{BS} / \mathrm{km}^{2}\right)$.

\section{A. Fundamentals of Average Performance Analysis}

From (18), (24), and (26), the covariance matrices of the four geolocation algorithms can be expressed as

$$
\mathbf{C}=\left(\mathbf{F}^{T} \mathbf{A F}\right)^{-1}
$$

where $\mathbf{A}$ is termed the information matrix. As defined in Section II, we have that for each geolocation algorithm

$$
\begin{aligned}
\mathbf{A}_{\mathrm{st}} & =\mathbf{R}^{-1} \\
\mathbf{A}_{\mathrm{s} \overline{\mathrm{t}}} & =\mathbf{H}^{T}\left(\mathbf{H R H} \mathbf{H}^{T}\right)^{-1} \mathbf{H} \\
\mathbf{A}_{\mathrm{hc}} & =\mathbf{A}_{\mathrm{s} \overline{\mathrm{t}}} \\
\mathbf{A}_{\mathrm{h} \overline{\mathrm{c}}} & =\mathbf{H}^{T}\left[\left(\mathbf{H R} \mathbf{H}^{T}\right) \odot \mathbf{I}\right]^{-1} \mathbf{H} .
\end{aligned}
$$

In (48), geometrical information is included in matrix $\mathbf{F}$ and in the noise power distribution defined by the measurement covariance matrix $\mathbf{R}$. From (13), the rows of $\mathbf{F}$ constitute the directive cosines of the vectors aligned with the paths from the mobile to the base stations (or generic references) with respect to the coordinate axes. Then

$$
\mathbf{F}=\mathbf{F}(\boldsymbol{\alpha})=\frac{1}{c}\left[\begin{array}{ll}
\cos \left(\alpha_{1}\right) & \sin \left(\alpha_{1}\right) \\
\cos \left(\alpha_{2}\right) & \sin \left(\alpha_{2}\right) \\
\cos \left(\alpha_{N}\right) & \sin \left(\alpha_{N}\right)
\end{array}\right]
$$

with $\boldsymbol{\alpha}=\left[\alpha_{1}, \alpha_{2}, \ldots, \alpha_{N}\right]^{T}$, and $\alpha_{n}$ the angle with respect to the horizontal coordinate axis of the vector from the mobile to the $n$th BS. The diagonal of $\mathbf{R}$ contains the noise power of all individual pseudo-TOA measurements, which depends mainly on the distance between the mobile and each BS. So, the specific position of the BSs affects the covariance matrix $\mathbf{C}$ in two different ways: in their angular distribution implicit in $\mathbf{F}$, and in their distance distribution implicit in $\mathbf{R}$. Hence, the geometry-independent average of the position error covariance matrix can be expressed as

$$
\overline{\mathbf{C}}=E_{\mathcal{G}}\left[\left(\mathbf{F}^{T} \mathbf{A F}\right)^{-1}\right]=E_{\mathcal{N}} E_{\boldsymbol{\alpha}}\left[\left(\mathbf{F}^{T} \mathbf{A F}\right)^{-1}\right]
$$

with $E_{\mathcal{G}}$ the expectation operator with respect to all possible geometry scenarios, which can be split into the expected value with respect to the angles $\boldsymbol{\alpha},\left(E_{\boldsymbol{\alpha}}\right)$, and the expected value with respect to the power distribution $\left(E_{\mathcal{N}}\right)$ (related with the distance distribution). Its analytical computation is too involved due to the particular way in which geometry affects the covariance structure. So, the expression derived in the sequel exploits a rotational property of the 2 -D location case in such a way that the structure of (52) can be studied without analytically computing its expected value.

For convenience, let us define the expectation with respect to the angles in (52) as $\overline{\mathbf{C}_{\mathcal{N}}}$

$$
\overline{\mathbf{C}}=E_{\mathcal{N}} \overline{\mathbf{C}_{\mathcal{N}}}
$$

where

$$
\overline{\mathbf{C}_{\mathcal{N}}}=E_{\boldsymbol{\alpha}}\left[\left(\mathbf{F}^{T} \mathbf{A F}\right)^{-1}\right]
$$

Here, the angles $\boldsymbol{\alpha}$ are independent, uniformly distributed random variables in $[0,2 \pi]$ due to the uniform random placement of BSs in the coverage area of the mobile. Thus, the infinite set of all possible angular configurations of the BSs can be divided into an infinite number of subsets within which angular configurations differ in a rotation. That is

$$
\left[\alpha_{1}, \alpha_{2}, \ldots, \alpha_{N}\right]=\alpha_{1} \cdot \mathbf{1}^{T}+\left[0, \alpha_{2}-\alpha_{1}, \ldots, \alpha_{N}-\alpha_{1}\right]
$$

with the rotated angles $\alpha_{i}-\alpha_{1}, 2 \leq i \leq N$ a set of mutually independent random variables ${ }^{2}$ uniformly distributed in $[0,2 \pi]$ and also independent of the equally uniformly distributed leading angle $\alpha_{1}$. Then, the expectation $\left(E_{\alpha}\right)$ in (54) can be expressed as the composition of two expectations: the expectation over the rotation $\alpha_{1}$ applied to the expectation over the subset of rotated angles $\mathcal{S} \mathcal{S}=\left\{\alpha_{i}-\alpha_{1}, 2 \leq i \leq N\right\}$. From this division into subsets of the geometry, (54) becomes

$$
\overline{\mathbf{C}_{\mathcal{N}}}=E_{\mathcal{S S}} E_{\beta}\left[\left(\mathbf{F}_{\mathcal{S}, \beta}^{T} \mathbf{A F}_{\mathcal{S S}, \beta}\right)^{-1}\right]
$$

where $\mathbf{F}_{\mathcal{S S}, \beta}$ denotes $\mathbf{F}$ for a particular rotation angle $\beta=\alpha_{1}$ and subset configuration. From (55), we can express the generic subset $\mathbf{F}_{\mathcal{S} S, \beta}^{T}$ matrix as

$$
\mathbf{F}_{\mathcal{S}, \beta}^{T}=\mathbf{G}_{\beta} \mathbf{F}_{\mathcal{S} \mathcal{S}}^{T}
$$

where $\mathbf{F}_{\mathcal{S} \mathcal{S}}^{T}=\mathbf{F}_{\mathcal{S} \mathcal{S}, 0}^{T}$ denotes the canonic $\mathbf{F}$ matrix of the subset in the specific case $\beta=0$, and $\mathbf{G}_{\beta}$ is a unitary-rotation matrix defined as

$$
\mathbf{G}_{\beta}=\left[\begin{array}{cc}
\cos (\beta) & \sin (\beta) \\
-\sin (\beta) & \cos (\beta)
\end{array}\right] .
$$

Now, applying (56)-(57), we have

$$
\overline{\mathbf{C}_{\mathcal{N}}}=E_{\mathcal{S S}} E_{\beta}\left[\left(\mathbf{G}_{\beta} \mathbf{F}_{\mathcal{S} \mathcal{S}}^{T} \mathbf{A} \mathbf{F}_{\mathcal{S S}} \mathbf{G}_{\beta}^{T}\right)^{-1}\right]
$$

and, as $\mathbf{G}_{\beta}$ is unitary

$$
\overline{\mathbf{C}_{\mathcal{N}}}=E_{\mathcal{S S}} E_{\beta}\left[\mathbf{G}_{\beta}\left(\mathbf{F}_{\mathcal{S} \mathcal{S}}^{T} \mathbf{A} \mathbf{F}_{\mathcal{S S}}\right)^{-1} \mathbf{G}_{\beta}^{T}\right]
$$

The expectation over $\beta$ is derived in Appendix C. Hence

$$
\overline{\mathbf{C}_{\mathcal{N}}}=\frac{1}{2} E_{\mathcal{S S}} \operatorname{tr}\left\{\left(\mathbf{F}_{\mathcal{S S}}^{T} \mathbf{A} \mathbf{F}_{\mathcal{S S}}\right)^{-1}\right\} \cdot \mathbf{I}
$$

\footnotetext{
${ }^{2}$ Note that the inherent wrapping operation in the modular difference of angles make these new random variables statistically independent.
} 
Although, in (60), the expectation over geometries is only partially solved, we may now see that only the average of a trace suffices to compute the geometry-independent performance of a location algorithm. It is also important to note from (53) and (60) that the average covariance matrix is always proportional to the identity matrix due to the rotational properties of the geolocation problem for uniformly distributed BSs. Similar results can be found in the case of a 3-D problem.

\section{B. Average Performance Analysis}

Applying (60) in (53) to the four algorithms associated with the information matrices $\mathbf{A}$ defined in (49), we obtain

$$
\begin{aligned}
\overline{\mathbf{C}}_{\mathrm{st}} & =\frac{1}{2} E_{\mathcal{N}, \mathcal{S S}} \operatorname{tr}\left\{\left(\mathbf{F}_{\mathcal{S} \mathcal{S}}^{T} \mathbf{R}^{-1} \mathbf{F}_{\mathcal{S S}}\right)^{-1}\right\} \cdot \mathbf{I} \\
\overline{\mathbf{C}}_{\mathrm{s} \overline{\mathrm{t}}} & =\frac{1}{2} E_{\mathcal{N}, \mathcal{S S}} \operatorname{tr}\left\{\left(\mathbf{F}_{\mathcal{S} \mathcal{S}}^{T} \mathbf{H}^{T}\left(\mathbf{H R H} \mathbf{H}^{T}\right)^{-1} \mathbf{H} \mathbf{F}_{\mathcal{S S}}\right)^{-1}\right\} \cdot \mathbf{I} \\
\overline{\mathbf{C}}_{\mathrm{hc}} & =\overline{\mathbf{C}}_{\mathrm{s} \overline{\mathrm{t}}} \\
\mathbf{C}_{\mathrm{h} \overline{\mathrm{c}}} & =\frac{1}{2} E_{\mathcal{N}, \mathcal{S} \mathcal{S}} \operatorname{tr}\left\{\left(\mathbf{F}_{\mathcal{S} \mathcal{S}}^{T} \mathbf{H}^{T}\left[\left(\mathbf{H R H} \mathbf{H}^{T}\right) \odot \mathbf{I}\right]^{-1} \mathbf{H} \mathbf{F}_{\mathcal{S S}}\right)^{-1}\right\} \cdot \mathbf{I}
\end{aligned}
$$

Performance analysis based on these expressions will be carried out in Section VII. The next section presents a lower bound to (61)-(64) to eliminate the $E_{\mathcal{S S}}$ operation.

\section{Lower Bound on the Average Covariance Matrix}

It has been shown in (53) that $\overline{\mathbf{C}}=E_{\mathcal{N}} \overline{\mathbf{C}_{\mathcal{N}}}$, where

$$
\overline{\mathbf{C}_{\mathcal{N}}}=E_{\alpha}\left[\left(\mathbf{F}^{T} \mathbf{A F}\right)^{-1}\right] \text {. }
$$

We can define the auxiliary term $\overline{\mathbf{C}_{\mathcal{N}}^{-1}}$ as

$$
\overline{\mathbf{C}_{\mathcal{N}}^{-1}}=E_{\alpha}\left[\left(\mathbf{F}^{T} \mathbf{A F}\right)\right]
$$

The advantadge of this new definition is that, as shown in Appendix G

$$
\overline{\mathbf{C}_{\mathcal{N}}}>\left[\overline{\mathbf{C}_{\mathcal{N}}^{-1}}\right]^{-1}
$$

and, as proved in Appendix $\mathrm{D}$, the new term $\overline{\mathbf{C}_{\mathcal{N}}^{-1}}$ presents a closed-form expression as

$$
\overline{\mathbf{C}_{\mathcal{N}}^{-1}}=\frac{1}{2 c^{2}} \operatorname{tr}\{\mathbf{A}\} \cdot \mathbf{I}
$$

So, from (53), (67), and (68), we have

$$
\overline{\mathbf{C}}>2 c^{2} E_{\mathcal{N}} \operatorname{tr}^{-1}\{\mathbf{A}\} \cdot \mathbf{I}
$$

which is a lower bound for all the covariance matrices associated with the four geolocation methods. Finally, from (69) and applying the information matrix definitions in (49), we can write the lower bound for the four geolocation algorithms as follows:

$$
\begin{aligned}
\overline{\mathbf{C}}_{\mathrm{st}} & >2 c^{2} E_{\mathcal{N}} \operatorname{tr}^{-1}\left\{\mathbf{R}^{-1}\right\} \cdot \mathbf{I} \\
\overline{\mathbf{C}}_{\mathrm{s} \overline{\mathrm{t}}} & \left.>2 c^{2} E_{\mathcal{N}} \operatorname{tr}^{-1}\left\{\mathbf{H}^{T}(\mathbf{H R H})^{T}\right)^{-1} \mathbf{H}\right\} \cdot \mathbf{I} \\
\overline{\mathbf{C}}_{\mathrm{hc}} & =\overline{\mathbf{C}}_{\mathrm{s} \overline{\mathrm{t}}} \\
\mathbf{C}_{\mathrm{h} \overline{\mathrm{c}}} & >2 c^{2} E_{\mathcal{N}} \operatorname{tr}^{-1}\left\{\mathbf{H}^{T}\left[\left(\mathbf{H R H} \mathbf{H}^{T}\right) \odot \mathbf{I}\right]^{-1} \mathbf{H}\right\} \cdot \mathbf{I} .
\end{aligned}
$$

The important remark in these expressions is that the angle distribution of the sources has been removed from the original exact expressions, while the expectation $E_{\mathcal{N}}$ over the noise power distribution remains.

One further approximation is possible for this lower bound: it is shown in Appendix E that for a large number of BSs, the random noise power distribution tends to be nonrandom if the components of the diagonal matrix $\mathbf{R}$ are sorted. It can also be easily shown that the order of the elements in $\mathbf{R}$ affects neither the performance of the circular algorithms nor the correlated version of the hyperbolic approach. In the particular case of the uncorrelated version of the hyperbolic approach, only the position of the reference BS affects the general performance of the algorithm. Then, the best and worst cases can be studied by assigning the smallest and the largest value of the sorted matrix $\mathbf{R}$ to the reference BS, respectively. Under this approximation, the $E_{\mathcal{N}}$ in expressions (70)-(72) can be removed if the random covariance matrix $\mathbf{R}$ is replaced by the nonrandom matrix $\tilde{\mathbf{R}}$. (See Appendix E for more details about the computation of $\tilde{\mathbf{R}}$ for a large number of BSs.) Finally, the following closed form expressions result:

$$
\begin{aligned}
\overline{\mathbf{C}}_{\mathrm{st}} & >2 c^{2} \operatorname{tr}^{-1}\left\{\tilde{\mathbf{R}}^{-1}\right\} \cdot \mathbf{I} \\
\overline{\mathbf{C}}_{\mathrm{s} \overline{\mathrm{t}}} & >2 c^{2} \operatorname{tr}^{-1}\left\{\mathbf{H}^{T}(\mathbf{H R} \mathbf{\mathbf { R }})^{-1} \mathbf{H}\right\} \cdot \mathbf{I} \\
\overline{\mathbf{C}}_{\mathrm{hc}} & =\overline{\mathbf{C}}_{\mathrm{s} \overline{\mathrm{t}}} \\
\mathbf{C}_{\mathrm{h} \overline{\mathrm{c}}} & \left.>2 c^{2} \operatorname{tr}^{-1}\left\{\mathbf{H}^{T}\left[\left(\mathbf{H} \tilde{\mathbf{R}} \mathbf{H}^{T}\right]\right) \odot \mathbf{I}\right]^{-1} \mathbf{H}\right\} \cdot \mathbf{I} .
\end{aligned}
$$

These expressions will be used only as approximations to the true simulations in Section VII.

\section{COMPARISON RESULTS}

\section{A. Base Stations Distribution}

One of the most important points in the evaluation of any location algorithm is the specific distribution of the BSs. In order to perform a fair comparison between methods, we will assume a completely random uniform distribution of the BSs in the visibility circle of the mobile. To see the effect of extremely bad dilution of precision conditions (i.e., unfortunate positioning of BSs), some histograms and cumulative histograms will be also presented.

In all the simulations presented here, it will be assumed that the mobile can observe BSs within a radius of $R \mathrm{~m}$ where BSs are uniformly distributed with a certain density of $\rho \mathrm{BS} / \mathrm{km}^{2}$. The effect of these two parameters will be studied in the simulations.

\section{B. Noise Profile}

The noise profile of time measurements is another important point in the evaluation of location algorithms. We have chosen the most common distribution presented in the literature based on real measurement campaigns.

Let $d$ be the distance from the mobile to a randomly selected beacon. Then, the probability density function (pdf) of $d$ is given by $p_{d}(d)=2 d / R^{2}$ for $0 \leq d \leq R$ [16]. On the other hand, the delay spread, which constitutes the principal phenomenon that 
disturbs the TOA measurements, is given by [16] as

$$
\sigma=T_{1} d^{\varepsilon} y
$$

where $T_{1}$ is the mean value of $\sigma$ at $d=1 \mathrm{~km}, \varepsilon$ is an exponent between 0.5 and 1.0, and $y$ is a log-normal random variable generated by $y=10^{Y / 10}$, where $Y$ is Gaussian with standard deviation $\sigma_{Y}$ in the interval between 2 and $6 \mathrm{~dB}$.

The performance results shown hereafter assume that the TOA covariance matrix $\mathbf{R}$ is diagonal, i.e.,

$$
\mathbf{R}=\operatorname{diag}\left(\left[\sigma_{1}^{2}, \sigma_{2}^{2}, \ldots, \sigma_{N}^{2}\right]\right)
$$

with $\sigma_{n}$ samples of the random variable $\sigma$ modeled in (76) for the $n$th beacon.

In all the simulations presented here, $T_{1}, \varepsilon$, and $\sigma_{Y}$ of (76) have been chosen for the suburban environment following [16]. That is, $T_{1}=0.4 \mu, \varepsilon=0.5$, and $\sigma_{Y}=4 \mathrm{~dB}$.

\section{Numerical Simulations I}

In this section, the performance of the four geolocation algorithms defined in (61)-(64) is evaluated. As the strict equivalence between the hyperbolic correlated and circular with unknown clock approaches has been established, only one is tested under the label hyperbolic correlated. Then, the three algorithms to be evaluated are

1) circular with known clock (or simply circular);

2) hyperbolic correlated (or simply hyperbolic);

3) hyperbolic uncorrelated.

In the case of the uncorrelated hyperbolic approach, the order of the variances in the diagonal of $\mathbf{R}$ is relevant in the $E_{\mathcal{N}}$ expectation operation, as discussed in Section VI. In the simulations, the best and worst cases are tested, which correspond to choosing the BSs with minimum and maximum pseudo-TOA, respectively, as the reference BS used to compute the TDOAs.

The simulations shown in this section follow the random averaging process explained in Section VI, according to the scenario defined in Sections VII-A and B.

First, the average performance of the three algorithms, as in (61), (62), and (64), is compared. Note that as these covariance matrices are proportional to the identity matrix, only a scalar need be plotted. Two different comparative analyses have been selected: In Fig. 1, the mean performance comparison can be observed for different visibility ranges $R$ assuming a constant BS density $\rho=0.25 \mathrm{BS} / \mathrm{Km}^{2}$ (scenario A). On the other hand, Fig. 2 shows also the same comparison but for several BS density values assuming a constant visibility range $R=3 \mathrm{~km}$ (scenario $B$ ). Note that for a better understanding of these figures (and also in the subsequent ones), the actual value of $N$, corresponding to the total number of beacons used, is indicated at each point in the curves. The value of $N$ is that associated with the given BS density $\rho$ and visibility range $R$, in the sense that $N=\pi R^{2} \rho$. The comparison provided in Figs. 1 and 2 shows the evolution in performance of the algorithms with respected to improvements in mobile technology (the visibility range), or to improvements in the network (an enhanced density of references). Although both trends increase the number of available references to be used for positioning, the noise power distribution that corrupts the TOA

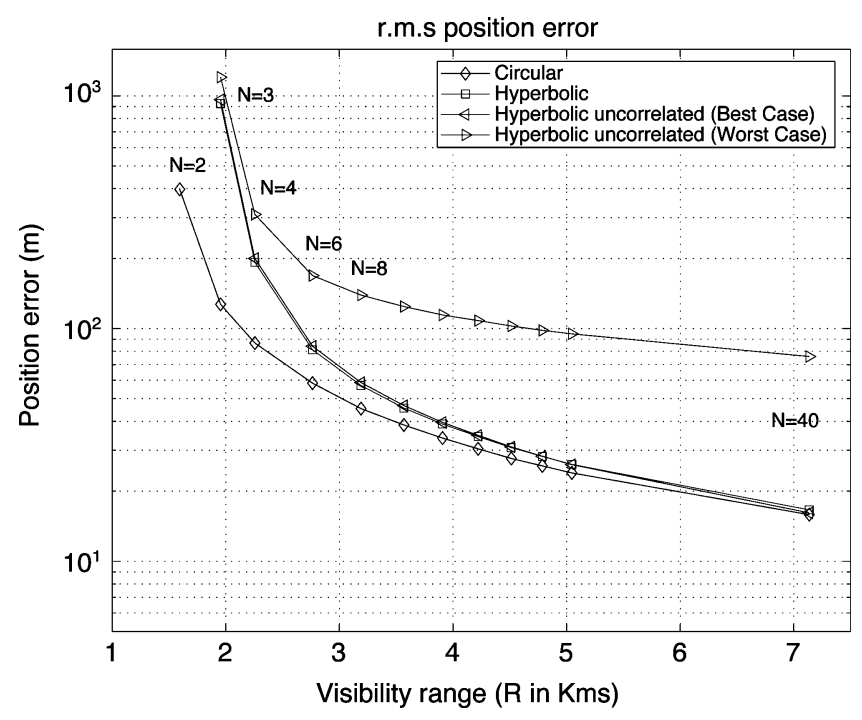

Fig. 1. Mean performance with a constant BS density of $\rho=0.25 \mathrm{BS} / \mathrm{km}^{2}$ (Scenario A).

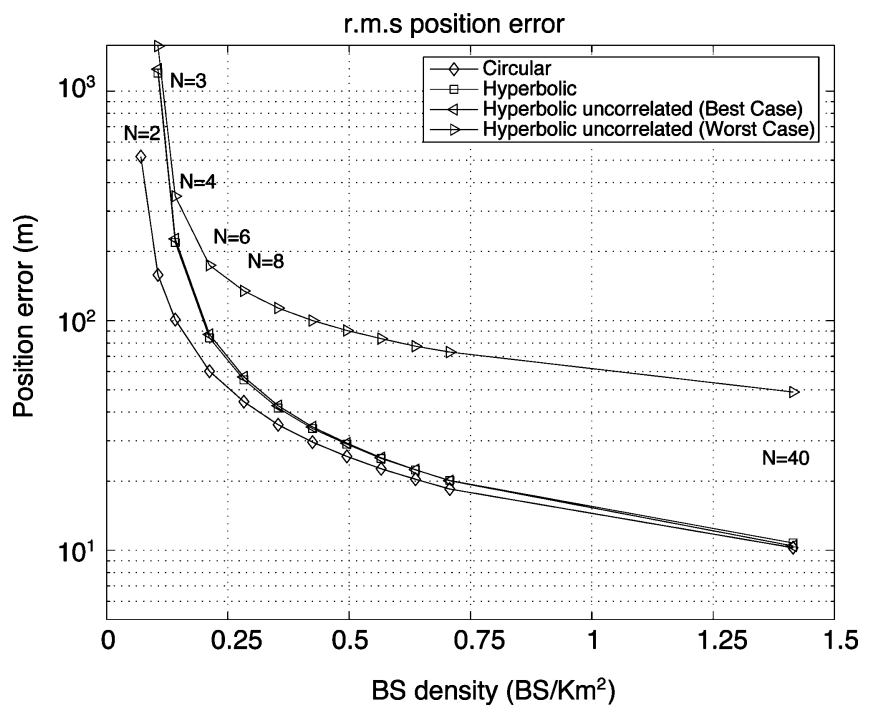

Fig. 2. Mean performance with a constant visibility range of $3 \mathrm{~km}$ (Scenario B).

measurements is to be reckoned with. This shows that location performance is more sensitive to enhanced network density than to enhanced mobile technology, although the available number of references can be the same.

In order to compare the mean performances with the theoretical limits, Figs. 3 and 4 show the ratio between the actual mean performances and the theoretical limits shown in (70), (71), and (72). Again, Fig. 3 shows this ratio for several values of the visibility range $R$ assuming a constant BS-density $\rho$ (scenario A), and Fig. 4 shows the ratio for several values of the BS density $\rho$ assuming a constant visibility range $R$ (scenario $\mathrm{B}$ ).

Finally, it is also interesting to compare the actual mean performance shown in Figs. 1 and 2 with respect to the approximate lower bounds presented in (73)-(75) analytically computed using fixed noise profiles. This comparison is presented in Fig. 5 for scenario A and Fig. 6 for scenario B. 


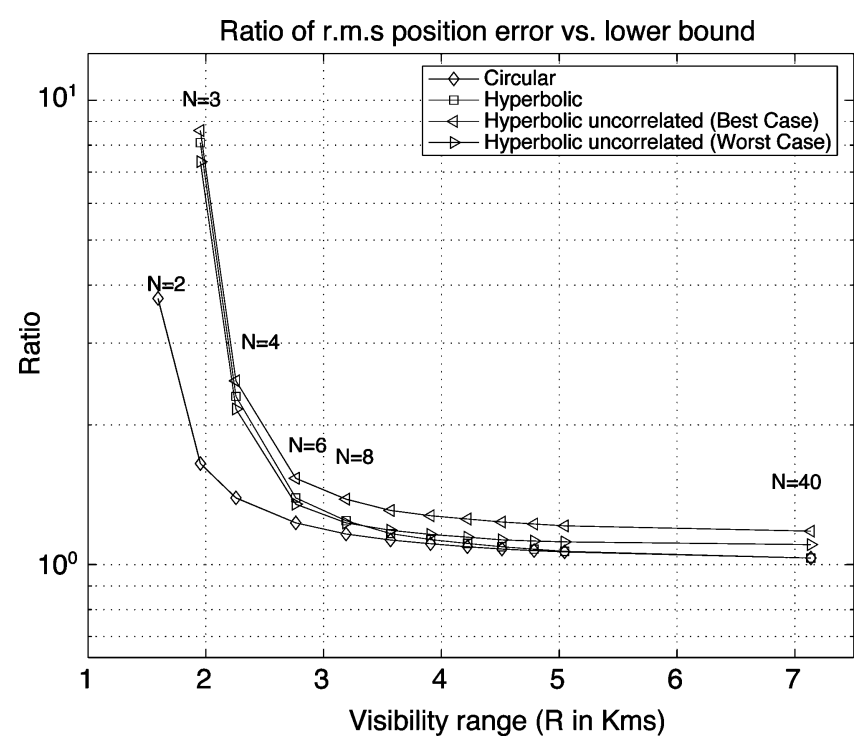

Fig. 3. Ratio between actual rms position error and its associated lower bound assuming a constant BS density of $\rho=0.25 \mathrm{BS} / \mathrm{km}^{2}$ (Scenario A).

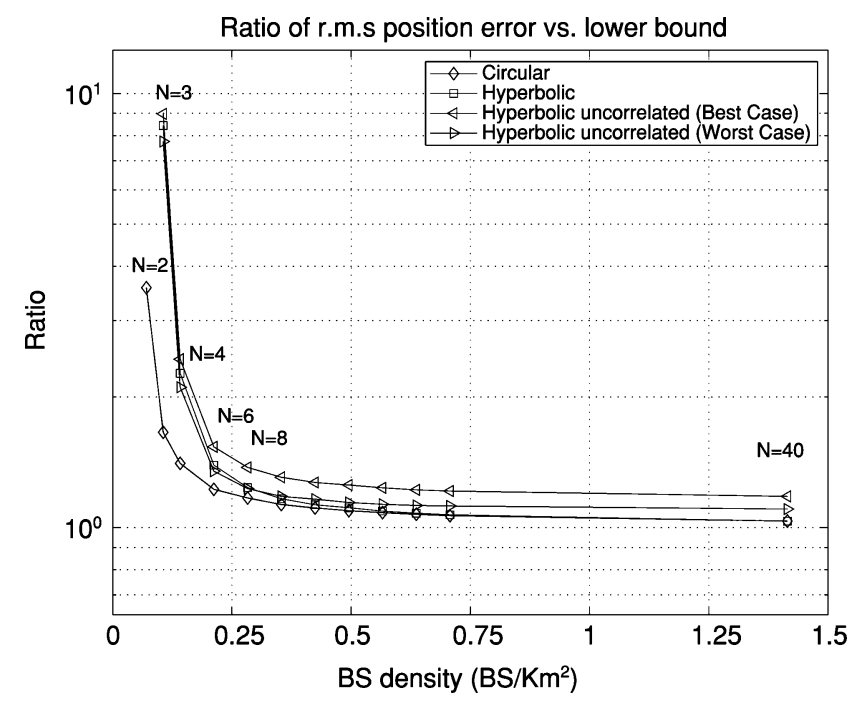

Fig. 4. Ratio between actual $\mathrm{rms}$ position error and its associated lower bound assuming a constant visibility range of $3 \mathrm{~km}$ (Scenario B).

\section{Numerical Simulations II}

The next suite of simulations shows the different performances achieved by a certain algorithm with a different number of BSs and the cumulative histograms of the mean position error. These simulations allow us to show the sensitivity of all the presented approaches to a bad dilution of precision (bad distribution of BSs) for a particular number of BSs.

First, the histogram and the cumulative histogram of the position error is studied. As results are quite similar for the four approaches, only the circular with known clock case is shown. In Figs. 7 and 8, the cumulative histogram and the histogram of the root mean square (rms) position error with different number of BSs can be observed, respectively.

Finally, in Figs. 9 and 10, the performances of the different approaches are compared in terms of its histogram and cumula-

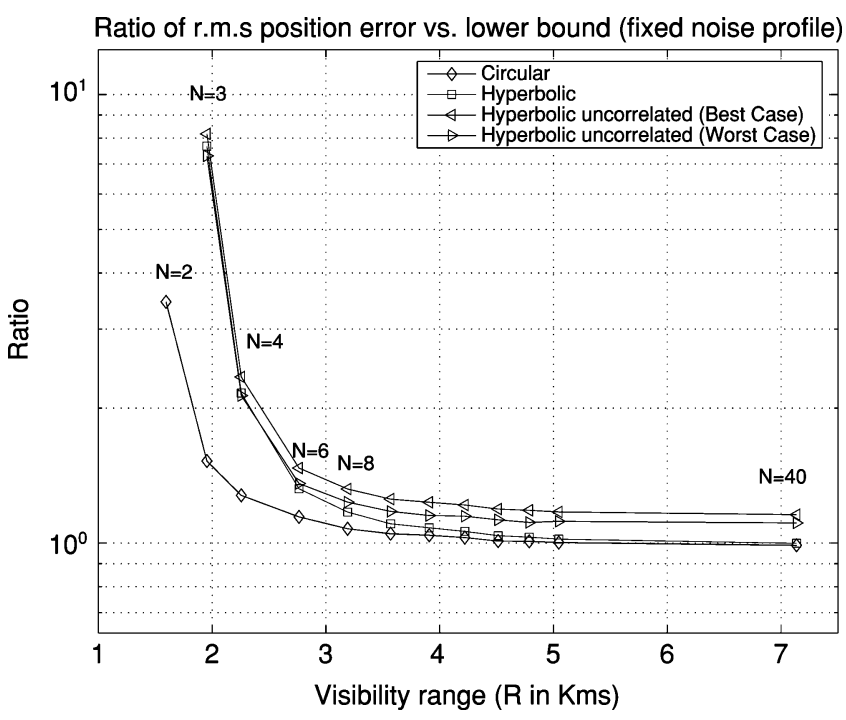

Fig. 5. Ratio between actual rms position error and its associated approximate lower bound (fixed noise profile) assuming a constant BS-density of $\rho=$ $0.25 \mathrm{BS} / \mathrm{km}^{2}$ (Scenario A).

Ratio of r.m.s position error vs. lower bound (fixed noise profile)

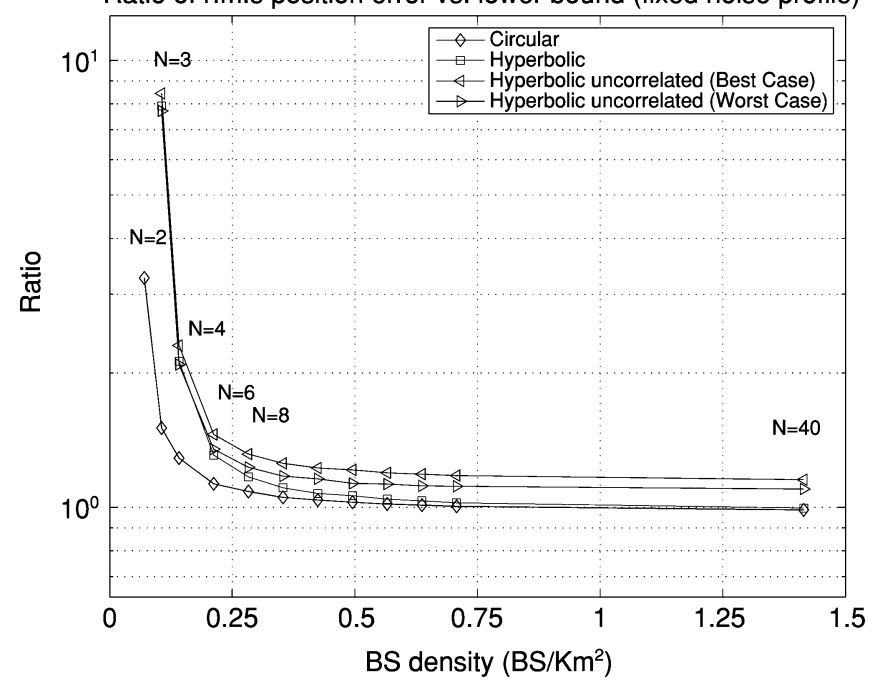

Fig. 6. Ratio between actual rms position error and its associated approximate lower bound (fixed noise profile) assuming a constant visibility range of $3 \mathrm{~km}$ (Scenario B).

tive histogram for a fixed number of BSs $(=5)$ in scenario with a density of $\rho=0.25 \mathrm{BS} / \mathrm{km}^{2}$. It can be observed that similar results are obtained for the correlated hyperbolic, and the best case of the hyperbolic (uncorrelated).

\section{E. Complexity}

We provide a brief sketch on complexity and latency issues for the four geolocation methods analyzed, wherein the relative computational cost between two methods may be defined in terms of the required number of multiplications and additions to deliver a position estimate from the TOA or TDOA measurements, at a fixed number of position estimates per second. On these terms, circular and hyperbolic locations present similar and reduced complexity as, in fact, they are based on a linearized weighted least squares solution of an error equation 


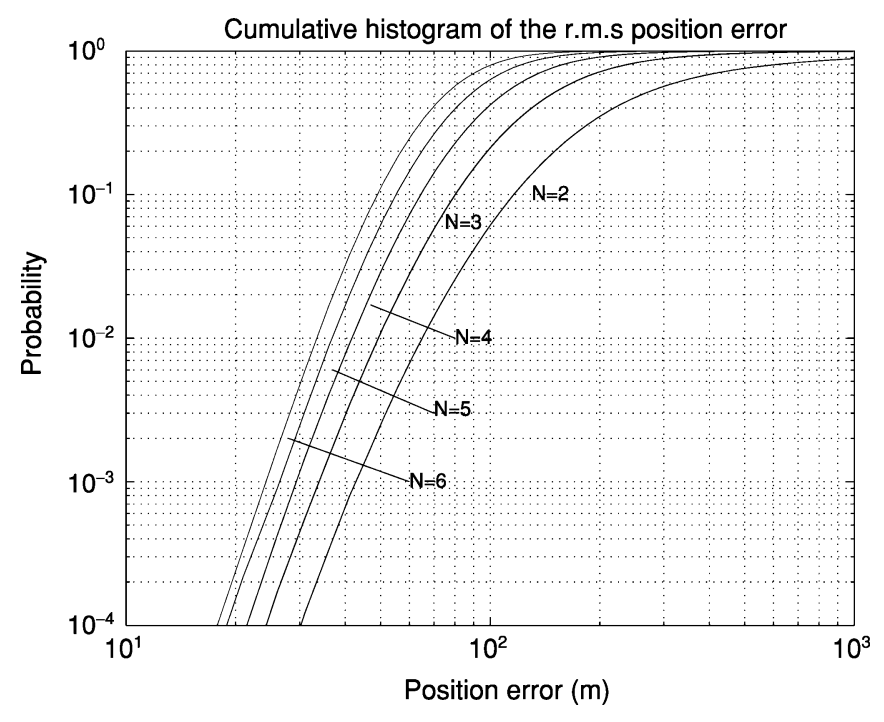

Fig. 7. Cumulative histogram of position error in the circular approach.

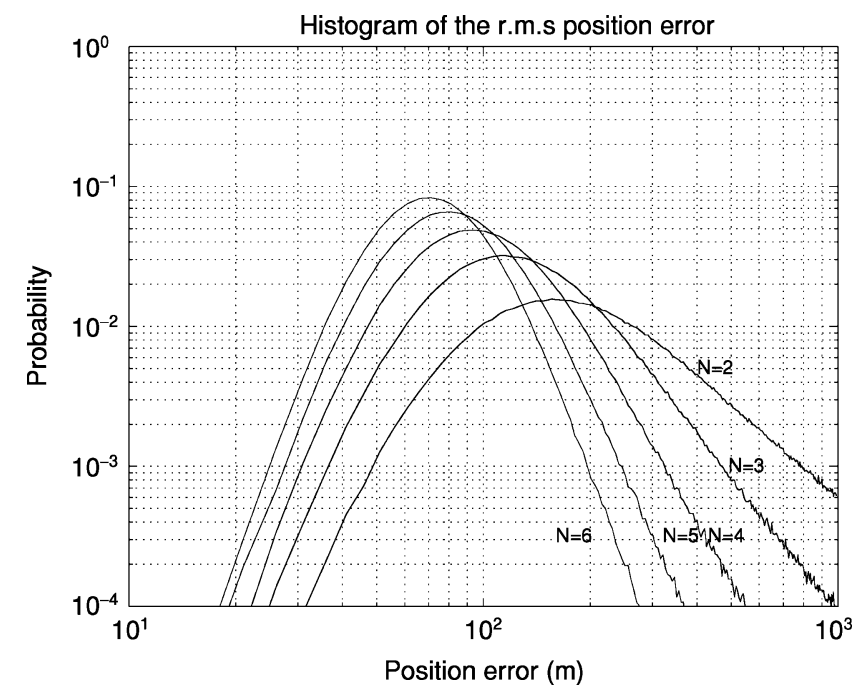

Fig. 8. Histogram of position error in the circular approach.

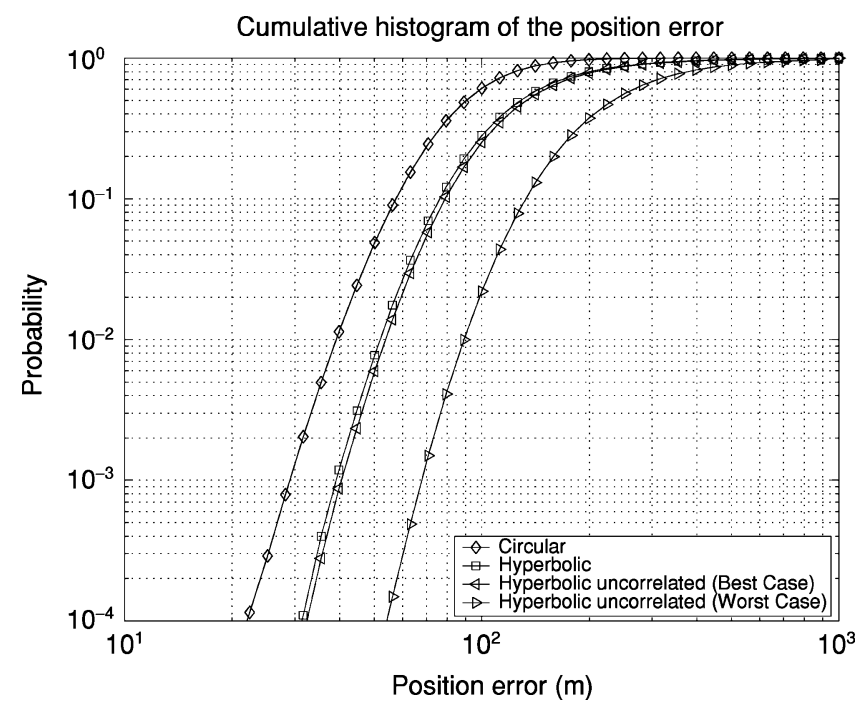

Fig. 9. Comparison between cumulative histograms of the position error using five base stations $(N=5)$.

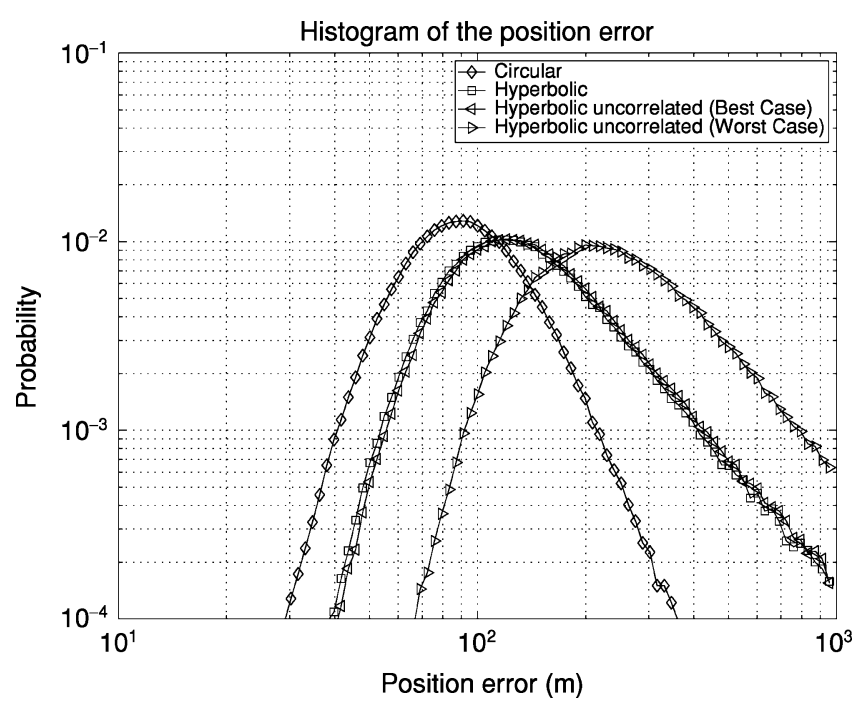

Fig. 10. Comparison between histograms of the position error using five base stations $(N=5)$.

[see (2) and (4)], which is endorsed by numerous publications on TOA and TDOA methods [19]-[24].

Two stages are identified: 1) a matrix vector product that computes the position estimate $\Delta \hat{\mathrm{x}}$ from the measurements $\Delta \hat{\mathrm{x}}$ (or $\Delta \hat{\mathbf{t}})$ as $\Delta \hat{\mathbf{x}}=\mathbf{W} \Delta \hat{\mathbf{t}}$, with matrix $\mathbf{W}$ defined from (16) and (21) and 2) the previous computation of matrix $\mathbf{W}$, involving the efficient evaluation of a matrix inverse. Due to the dependence of $\mathbf{W}$ on $\mathbf{F}$ (geometry matrix), $\mathbf{W}$ is not generally a sparse matrix, so that the complexity of the 1) stage is practically equal for the four methods (taking into account that for circular and hyperbolic methods, $N$ TOA and $N-1$ TDOA measurements are required, respectively). The computation of the estimation matrix $\mathbf{W}$ is very similar for the four methods. For hyperbolic methods, more additions are necessary to perform matrix multiplication by the sparse $\mathbf{H}$ (TOA difference) matrix and, in particular, the correlated hyperbolic method requires inversion of the sparse correlation matrix $\mathbf{R}_{\mathrm{h}}$ (10).

We should also be reminded that the circular with known or unknown clock and correlated hyperbolic methods are capable of processing simultaneous timing measurements, which, in a possible CDMA scenario, requires parallel despreading of signals from different BSs. In this sense, the uncorrelated hyperbolic method is less demanding of hardware as timing measurements are obtained sequentially. Consequenltly, latency (excluding computational delays of the operation $\Delta \hat{\mathbf{x}}=\mathbf{W} \Delta \hat{\mathbf{t}}$ ) is higher for the uncorrelated hyperbolic method, in contrast to the other three methods where all TOA measurements are performed simultaneously.

\section{CONCLUSION}

A comparative analysis of the accuracy of various location techniques has been performed. Four representative schemes have been identified: the circular (TOA-based) approach with and without knowledge of the receiver clock and the hyperbolic approach, computing the TDOAs at the same or at different time instants. 
Strictly equal performance has been shown for the "circular unknown clock" and the "hyperbolic correlated" methods. This statement is general, with independence of the statistical properties of the error sources affecting the measurements, and of the beacon pairs used to estimate each TDOA. This is a generalization of some previous work for uncorrelated errors of equal variance. It has also been shown that the "circular known clock" method is strictly better than the two other methods, in a measure defined by the specific configuration of the beacons.

We have also analyzed the expected location accuracy averaged over an ensemble of realistic random geometries. First, it has been found that the "circular unknown clock" and "hyperbolic correlated" methods yield an average performance that asymptotically approaches that of the best "circular known clock" method, when the number of beacons is high. That is, the knowledge of the receiver clock yields significant improvement of the average accuracy only when a small number of beacons is used. Second, average analysis applied to the "hyperbolic uncorrelated" case has shown this method to yield the worstcase average performance and, contrary to the other methods, it proves very sensitive to the selection of the beacon pairs used to compute the TDOAs, especially when the number of available beacons is high. Best performance is obtained for the "hyperbolic uncorrelated" method if the beacon associated with the minimum measurement error is chosen as a reference. In conclusion, our analysis shows that for the hyperbolic uncorrelated method, the reference beacon should always be chosen as that with the minimum timing error variance (best case) if optimum performance is to be achieved. To determine the criticality of this choice, the worst case (the reference beacon has maximum timing error variance) has also been evaluated. In the simulation results, the gap between the best and worst case curves indicates how critical the correct selection of the best beacon is. The impact of transferring to a new reference beacon is negligible in terms of computational complexity, because it only determines the inputs to the algorithm, although it may lead to nonnegligible hardware complexity if a specific best-beacon selection mechanism in terms of the timing error variance of the measurements were to be designed. It is interesting to note that this mechanism could instead be based on the natural handover mechanism implemented in the mobile station. We should also be aware that the selection of the most powerful signal (associated with the handover process) should be highly (although not exactly) correlated with the minimum timing error variance. For this reason, if in the hyperbolic uncorrelated method the reference beacon used for the TDOA computation is the reference station used in the handover process, the actual performance will be close, in practice, to the best case curves.

These methods have been evaluated for realistic propagation channel models characterizing the delay spread associated with different environment conditions. For each method, we have analyzed the effect of increasing the BS density and the effect of enhancing the mobile terminal hearability of distant basestations. The former is shown to yield slightly better results than the latter.

Finally, this paper has derived a performance lower bound for the average performance of location techniques, which proves to be very accurate for a sufficiently high number of beacons. Future work will focus on the derivation of more accurate performance bounds that are valid for any number of beacons.

\section{APPENDIX A}

\section{COVARIANCE OF HyPERBOLIC METHOdS}

In this Appendix, we examine the relationship between the covariance matrices $\mathbf{C}_{\mathrm{hc}}=\mathbf{C}_{\mathrm{s} \overline{\mathrm{t}}}$ and $\mathbf{C}_{\mathrm{h} \overline{\mathrm{c}}}$ in (17) and (24), respectively:

$$
\begin{aligned}
& \mathbf{C}_{\mathrm{hc}}=\mathbf{T}^{T}\left(\mathbf{F}_{1}^{T} \mathbf{R}^{-1} \mathbf{F}_{1}\right)^{-1} \mathbf{T} \\
& \mathbf{C}_{\mathrm{h} \bar{c}}=\left[\mathbf{F}^{T}\left(\mathbf{H}^{T} \mathbf{R}_{\mathrm{h} \bar{c}}^{-1} \mathbf{H}\right) \mathbf{F}\right]^{-1}
\end{aligned}
$$

From (30), (32), and (37), we have

$$
\begin{aligned}
\mathbf{C}_{\mathrm{hc}}^{-1} & =\mathbf{C}_{\mathrm{st}}^{-1}=\mathbf{F}^{T}\left(\mathbf{R}^{-1}-\frac{\mathbf{R}^{-1} \mathbf{1} \mathbf{1}^{T} \mathbf{R}^{-1}}{\mathbf{1}^{T} \mathbf{R}^{-1} \mathbf{1}}\right) \mathbf{F} \\
& =\frac{1}{\sigma^{2}} \mathbf{F}^{T}\left(\mathbf{D}_{\mathrm{c}}^{-1}-\frac{\mathbf{D}_{\mathrm{c}}^{-1} \mathbf{1}^{T} \mathbf{D}_{\mathrm{c}}^{-1}}{\mathbf{1}^{T} \mathbf{D}_{\mathrm{c}}^{-1} \mathbf{1}}\right) \mathbf{F} \\
& =\frac{1}{\sigma^{2}} \mathbf{F}^{T} \mathbf{D}_{\mathrm{c}}^{-1 / 2}\left(\mathbf{I}-\frac{\mathbf{D}_{\mathrm{c}}^{-1 / 2} \mathbf{1 1}^{T} \mathbf{D}_{\mathrm{c}}^{-1 / 2}}{\mathbf{1}^{T} \mathbf{D}_{\mathrm{c}}^{-1 / 2} \mathbf{D}_{\mathrm{c}}^{-1 / 2} \mathbf{1}}\right) \mathbf{D}_{\mathrm{c}}^{-1 / 2} \mathbf{F} .
\end{aligned}
$$

Now, we let

$$
\mathbf{F}_{\mathrm{c}}=\mathbf{D}_{\mathrm{c}}^{-1 / 2} \mathbf{F}, \quad \mathbf{a}_{\mathrm{c}}=\mathbf{D}_{\mathrm{c}}^{-1 / 2} \mathbf{1}
$$

Hence

$$
\mathbf{C}_{\mathrm{hc}}^{-1}=\frac{1}{\sigma^{2}} \mathbf{F}_{\mathrm{c}}^{T}\left(\mathbf{I}-\frac{\mathbf{a}_{\mathrm{c}} \mathbf{a}_{\mathrm{c}}^{T}}{\mathbf{a}_{\mathrm{c}}^{T} \mathbf{a}_{\mathrm{c}}}\right) \mathbf{F}_{\mathrm{c}} .
$$

We now consider the second covariance matrix $\mathbf{C}_{\mathrm{h} \bar{c}}$. From (24) and (38), we have

$$
\begin{aligned}
\mathbf{C}_{\mathrm{h} \overline{\mathrm{c}}}^{-1} & =\mathbf{F}^{T}\left(\mathbf{H}^{T} \mathbf{R}_{\mathrm{h} \overline{\mathrm{c}}}^{-1} \mathbf{H}\right) \mathbf{F} \\
& =\frac{1}{\sigma_{\mathrm{h} \overline{\mathrm{c}}}^{2}} \mathbf{F}^{T}\left(\mathbf{H}^{T} \mathbf{D}_{\overline{\mathrm{c}}}^{-1} \mathbf{H}\right) \mathbf{F} \\
& =\frac{1}{\sigma_{\mathrm{h} \overline{\mathrm{c}}}^{2}} \mathbf{F}_{\mathrm{c}}^{T} \mathbf{H}_{\overline{\mathrm{c}}}^{T} \mathbf{H}_{\overline{\mathrm{c}}} \mathbf{F}_{\mathrm{c}}
\end{aligned}
$$

where $\mathbf{F}_{\mathrm{c}}$ was defined in (79), and $\mathbf{H}_{\overline{\mathrm{c}}}$ is defined as $\mathbf{H}_{\overline{\mathrm{c}}}=$ $\mathbf{D}_{\overline{\mathrm{c}}}^{-1 / 2} \mathbf{H D}_{\mathrm{c}}^{1 / 2}$. Note that

$$
\mathbf{H}_{\overline{\mathrm{c}}} \mathbf{a}_{\mathrm{c}}=\mathbf{D}_{\overline{\mathrm{c}}}^{-1 / 2} \mathbf{H} \mathbf{1}=\mathbf{0} .
$$

Now, considering (80) and (83), we find that both inverse covariance matrices can be expressed as $\mathbf{F}_{\mathrm{c}}^{T} \mathbf{A} \mathbf{F}_{\mathrm{c}}$ for some matrix A. The respective matrices $\mathbf{A}$ fulfill the following properties (see proofs in Appendix B):

$$
\begin{aligned}
\operatorname{tr}\left(\mathbf{H}_{\overline{\mathrm{c}}}^{T} \mathbf{H}_{\overline{\mathrm{c}}}\right) & =\frac{\sigma_{\mathrm{h} \overline{\mathrm{c}}}^{2}}{\sigma^{2}} \operatorname{tr}\left(\mathbf{I}-\frac{\mathbf{a}_{\mathrm{c}} \mathbf{a}_{\mathrm{c}}^{T}}{\mathbf{a}_{\mathrm{c}}^{T} \mathbf{a}_{\mathrm{c}}}\right)=(N-1) \frac{\sigma_{\mathrm{h} \bar{c}}^{2}}{\sigma^{2}} \\
\operatorname{span}\left\langle\mathbf{H}_{\overline{\mathrm{c}}}^{T} \mathbf{H}_{\overline{\mathrm{c}}}\right\rangle & =\operatorname{span}\left\langle\mathbf{I}-\frac{\mathbf{a}_{\mathrm{c}} \mathbf{a}_{\mathrm{c}}^{T}}{\mathbf{a}_{\mathrm{c}}^{T} \mathbf{a}_{\mathrm{c}}}\right\rangle=\operatorname{span}\left\langle\mathbf{a}_{\mathrm{c}}\right\rangle_{\perp} \\
\mathbf{H}_{\overline{\mathrm{c}}} & =\mathbf{H}_{\overline{\mathrm{c}}}\left(\mathbf{I}-\frac{\mathbf{a}_{\mathrm{c}} \mathbf{a}_{\mathrm{c}}^{T}}{\mathbf{a}_{\mathrm{c}}^{T} \mathbf{a}_{\mathrm{c}}}\right) .
\end{aligned}
$$


Using these properties, to fulfill the trace constraint in (85), some eigenvalues of $\left(\sigma^{2} / \sigma_{\mathrm{h}}^{2}\right) \mathbf{H}_{\overline{\mathrm{c}}}^{T} \mathbf{H}_{\overline{\mathrm{c}}}$ need to be superior to 1 , and some need be inferior (note that all the nonzero eigenvalues of $\left(\mathbf{I}-\left(\mathbf{a}_{\mathrm{c}} \mathbf{a}_{\mathrm{c}}^{T} / \mathbf{a}_{\mathrm{c}}^{T} \mathbf{a}_{\mathrm{c}}\right)\right)$ are unitary $)$. We also have

$$
\frac{1}{\sigma_{\mathrm{h} \overline{\mathrm{c}}}^{2}} \mathbf{H}_{\overline{\mathrm{c}}}^{T} \mathbf{H}_{\overline{\mathrm{c}}}=\frac{1}{\sigma_{\mathrm{h} \overline{\mathrm{c}}}^{2}}\left(\mathbf{I}-\frac{\mathbf{a}_{\mathrm{c}} \mathbf{a}_{\mathrm{c}}^{T}}{\mathbf{a}_{\mathrm{c}}^{T} \mathbf{a}_{\mathrm{c}}}\right) \mathbf{H}_{\overline{\mathrm{c}}}^{T} \mathbf{H}_{\overline{\mathrm{c}}}\left(\mathbf{I}-\frac{\mathbf{a}_{\mathrm{c}} \mathbf{a}_{\mathrm{c}}^{T}}{\mathbf{a}_{\mathrm{c}}^{T} \mathbf{a}_{\mathrm{c}}}\right) .
$$

Hence, from (83), $\mathbf{C}_{\mathrm{h} \overline{\mathrm{c}}}^{-1}$ can be expressed as

$$
\mathbf{C}_{\mathrm{h} \overline{\mathrm{c}}}^{-1}=\mathbf{F}_{\mathrm{c}}^{T} \frac{1}{\sigma_{\mathrm{h} \overline{\mathrm{c}}}^{2}}\left(\mathbf{I}-\frac{\mathbf{a}_{\mathrm{c}} \mathbf{a}_{\mathrm{c}}^{T}}{\mathbf{a}_{\mathrm{c}}^{T} \mathbf{a}_{\mathrm{c}}}\right) \mathbf{H}_{\overline{\mathrm{c}}}^{T} \mathbf{H}_{\overline{\mathrm{c}}}\left(\mathbf{I}-\frac{\mathbf{a}_{\mathrm{c}} \mathbf{a}_{\mathrm{c}}^{T}}{\mathbf{a}_{\mathrm{c}}^{T} \mathbf{a}_{\mathrm{c}}}\right) \mathbf{H D}_{\mathrm{c}}
$$

In turn, from (80), and using the idempotent property of projection operators, $\mathbf{C}_{\mathrm{hc}}^{-1}$ can be expressed as

$$
\mathbf{C}_{\mathrm{hc}}^{-1}=\mathbf{F}_{\mathrm{c}}^{T} \frac{1}{\sigma^{2}}\left(\mathbf{I}-\frac{\mathbf{a}_{\mathrm{c}} \mathbf{a}_{\mathrm{c}}^{T}}{\mathbf{a}_{\mathrm{c}}^{T} \mathbf{a}_{\mathrm{c}}}\right)\left(\mathbf{I}-\frac{\mathbf{a}_{\mathrm{c}} \mathbf{a}_{\mathrm{c}}^{T}}{\mathbf{a}_{\mathrm{c}}^{T} \mathbf{a}_{\mathrm{c}}}\right) \mathbf{F}_{\mathrm{c}}
$$

So by setting $\mathbf{F}_{\mathrm{c}, \mathrm{a}}=\left(\mathbf{I}-\left(\mathbf{a}_{\mathrm{c}} \mathbf{a}_{\mathrm{c}}^{T} / \mathbf{a}_{\mathrm{c}}^{T} \mathbf{a}_{\mathrm{c}}\right)\right) \mathbf{F}_{\mathrm{c}}$, we have

$$
\begin{aligned}
\mathbf{C}_{\mathrm{hc}}^{-1} & =\mathbf{F}_{\mathrm{c}, \mathrm{a}}^{T} \frac{\mathbf{I}}{\sigma^{2}} \mathbf{F}_{\mathrm{c}, \mathrm{a}} \\
\mathbf{C}_{\mathrm{h} \overline{\mathrm{c}}}^{-1} & =\mathbf{F}_{\mathrm{c}, \mathrm{a}}^{T}\left(\frac{1}{\sigma_{\mathrm{h} \overline{\mathrm{c}}}^{2}} \mathbf{H}_{\overline{\mathrm{c}}}^{T} \mathbf{H}_{\overline{\mathrm{c}}}\right) \mathbf{F}_{\mathrm{c}, \mathrm{a}} .
\end{aligned}
$$

Now, from the eigenvalue decomposition $\mathbf{H}_{\overline{\mathrm{c}}}^{T} \mathbf{H}_{\overline{\mathrm{c}}}=\mathbf{U} \boldsymbol{\Lambda} \mathbf{U}^{T}$, with $\mathbf{U}$ a unitary matrix $\left(\mathbf{U U}^{T}=\mathbf{I}\right)$ and $\boldsymbol{\Lambda}$ diagonal, and taking $\mathbf{F}_{\mathrm{u}}=(1 / \sigma) \mathbf{U}^{T} \mathbf{F}_{\mathrm{c}, \mathrm{a}}$, we can recast (88) into

$$
\mathbf{C}_{\mathrm{hc}}^{-1}=\mathbf{F}_{\mathrm{u}}^{T} \mathbf{F}_{\mathrm{u}}, \quad \mathbf{C}_{\mathrm{h} \overline{\mathrm{c}}}^{-1}=\mathbf{F}_{\mathrm{u}}^{T}\left(\frac{\sigma^{2}}{\sigma_{\mathrm{h} \overline{\mathrm{c}}}^{2}} \boldsymbol{\Lambda}\right) \mathbf{F}_{\mathrm{u}}
$$

where $\mathbf{F}_{\mathrm{u}}=\mathbf{W}^{T} \mathbf{F}$, with $\mathbf{W}=(1 / \sigma) \mathbf{U}^{T}\left(\mathbf{I}-\left(\mathbf{a}_{\mathrm{c}} \mathbf{a}_{\mathrm{c}}^{T} / \mathbf{a}_{\mathrm{c}}^{T} \mathbf{a}_{\mathrm{c}}\right)\right)$ $\mathbf{D}_{\mathrm{c}}^{-1 / 2}$. Hence, the relationship between the covariance of both methods is obtained, where using $\operatorname{tr}\left(\mathbf{H}_{\overline{\mathrm{C}}}^{T} \mathbf{H}_{\overline{\mathrm{C}}}\right)=\operatorname{tr}(\boldsymbol{\Lambda})$, and the property in (85) yields

$$
\frac{\sigma^{2}}{\sigma_{\mathrm{h} \overline{\mathrm{c}}}^{2}} \operatorname{tr}(\boldsymbol{\Lambda})=N-1 .
$$

\section{APPENDIX B}

\section{PROPERTIES OF $\mathbf{H}_{\bar{c}}$}

We will first evaluate the trace of $\mathbf{H}_{\overline{\mathrm{c}}}^{T} \mathbf{H}_{\overline{\mathrm{c}}}$. The proof is simple but lengthy. Using the definition of $\mathbf{H}_{\bar{c}}$ and the trace property $\operatorname{tr}(\mathbf{A B})=\operatorname{tr}(\mathbf{B A})$, we have

$$
\begin{aligned}
\operatorname{tr}\left(\mathbf{H}_{\overline{\mathrm{c}}}^{T} \mathbf{H}_{\overline{\mathrm{c}}}\right) & =\operatorname{tr}\left(\left(\mathbf{D}_{\overline{\mathrm{c}}}^{-1 / 2} \mathbf{H} \mathbf{D}_{\mathrm{c}}^{1 / 2}\right)^{T} \mathbf{D}_{\overline{\mathrm{c}}}^{-1 / 2} \mathbf{H} \mathbf{D}_{\mathrm{c}}^{1 / 2}\right) \\
& =\operatorname{tr}\left(\mathbf{D}_{\mathrm{c}}^{1 / 2} \mathbf{H}^{T} \mathbf{D}_{\overline{\mathrm{c}}}^{-1 / 2} \mathbf{D}_{\overline{\mathrm{c}}}^{-1 / 2} \mathbf{H} \mathbf{D}_{\mathrm{c}}^{1 / 2}\right) \\
& =\operatorname{tr}\left(\mathbf{H}^{T} \mathbf{D}_{\overline{\mathrm{c}}}^{-1} \mathbf{H} \mathbf{D}_{\mathrm{c}}\right) .
\end{aligned}
$$

We proceed to evaluate the matrix product within the trace for $\mathbf{H}=[\mathbf{1},-\mathbf{I}]$ using the definitions in (37)-(40). Only nonzero components are shown:

$\mathbf{D}_{\overline{\mathrm{c}}}^{-1} \mathbf{H D}_{\mathrm{c}}$

$$
=\frac{\sigma_{\mathrm{h} \overline{\mathrm{c}}}^{2}}{\sigma^{2}}\left[\begin{array}{cccc}
\frac{s_{1}^{2}}{s_{1}^{2}+s_{2}^{2}} & -\frac{s_{2}^{2}}{s_{1}^{2}+s_{2}^{2}} & & \\
\vdots & & \ddots & \\
\frac{s_{1}^{2}}{s_{1}^{2}+s_{N}^{2}} & & & -\frac{s_{N}^{2}}{s_{1}^{2}+s_{N}^{2}}
\end{array}\right]
$$

$\operatorname{diag}\left(\mathbf{H}^{T} \mathbf{D}_{\overline{\mathrm{c}}}^{-1}\right.$

$$
=\frac{\sigma_{\mathrm{h} \overline{\mathrm{c}}}^{2}}{\sigma^{2}}\left[s_{1}^{2} \sum_{l=1}^{N-1} \frac{1}{s_{1}^{2}+s_{l+1}^{2}}, \frac{s_{2}^{2}}{s_{1}^{2}+s_{2}^{2}}, \ldots, \frac{s_{N}^{2}}{s_{1}^{2}+s_{N}^{2}}\right]^{T} .
$$

Hence

$$
\begin{aligned}
\operatorname{tr} & \left(\mathbf{H}^{T} \mathbf{D}_{\overline{\mathrm{c}}}^{-1} \mathbf{H} \mathbf{D}_{\mathrm{c}}\right) \\
& =\frac{\sigma_{\mathrm{h} \bar{c}}^{2}}{\sigma^{2}}\left(\sum_{l=1}^{N-1} \frac{s_{1}^{2}}{s_{1}^{2}+s_{l+1}^{2}}+\sum_{l=1}^{N-1} \frac{s_{l+1}^{2}}{s_{1}^{2}+s_{l+1}^{2}}\right) \\
& =(N-1) \frac{\sigma_{\mathrm{h} \bar{c}}^{2}}{\sigma^{2}}
\end{aligned}
$$

and

$$
\operatorname{tr}\left(\mathbf{H}_{\overline{\mathrm{c}}}^{T} \mathbf{H}_{\overline{\mathrm{c}}}\right)=(N-1) \frac{\sigma_{\mathrm{h} \overline{\mathrm{c}}}^{2}}{\sigma^{2}}=\frac{\sigma_{\mathrm{h} \overline{\mathrm{c}}}^{2}}{\sigma^{2}} \operatorname{tr}\left(\mathbf{I}-\frac{\mathbf{a}_{\mathrm{c}} \mathbf{a}_{\mathrm{c}}^{T}}{\mathbf{a}_{\mathrm{c}}^{T} \mathbf{a}_{\mathrm{c}}}\right)
$$

as the eigenvalues of the projection operator $\left.\mathbf{I}-\left(\mathbf{a}_{\mathrm{c}} \mathbf{a}_{\mathrm{c}}^{T}\right) / \mathbf{a}_{\mathrm{c}}^{T} \mathbf{a}_{\mathrm{c}}\right)$ are $N-11$ s and one 0 . The subspace spanned by the rows of the following matrices is the same for the orthogonality property $\mathbf{H}_{\overline{\mathrm{c}}} \mathbf{a}_{\mathrm{c}}=\mathbf{0}$ in (84):

$$
\operatorname{span}\left\langle\mathbf{H}_{\overline{\mathrm{c}}}^{T} \mathbf{H}_{\overline{\mathrm{c}}}\right\rangle=\operatorname{span}\left\langle\mathbf{I}-\frac{\mathbf{a}_{\mathrm{c}} \mathbf{a}_{\mathrm{c}}^{T}}{\mathbf{a}_{\mathrm{c}}^{T} \mathbf{a}_{\mathrm{c}}}\right\rangle=\operatorname{span}\left\langle\mathbf{a}_{\mathrm{c}}\right\rangle_{\perp}
$$

hence we necessarily have

$$
\mathbf{H}_{\overline{\mathrm{c}}}=\mathbf{H}_{\overline{\mathrm{c}}}\left(\mathbf{I}-\frac{\mathbf{a}_{\mathrm{c}} \mathbf{a}_{\mathrm{c}}^{T}}{\mathbf{a}_{\mathrm{c}}^{T} \mathbf{a}_{\mathrm{c}}}\right)
$$

as $\mathbf{I}-\left(\mathbf{a}_{\mathrm{c}} \mathbf{a}_{\mathrm{c}}^{T} / \mathbf{a}_{\mathrm{c}}^{T} \mathbf{a}_{\mathrm{c}}\right)$ is the projector onto the subspace orthogonal to $\mathbf{a}_{\mathrm{c}}$.

\section{APPENDIX C}

\section{ROTATIONAL AVERAGING}

It is proved here that for symmetric matrix $\mathbf{A}$, it holds that

$$
\mathbf{B}=E_{\beta}\left[\mathbf{G}_{\beta} \mathbf{A G}_{\beta}^{T}\right]=\frac{1}{2} \operatorname{tr}\{\mathbf{A}\} \cdot \mathbf{I}
$$

where $E_{\beta}$ denotes the expectation operation over $\beta$. A is a $2 \times 2$ matrix

$$
\mathbf{A}=\left[\begin{array}{ll}
a_{11} & a_{12} \\
a_{21} & a_{22}
\end{array}\right]
$$

and $\mathbf{G}_{\beta}$ is the previously defined unitary rotation matrix

$$
\mathbf{G}_{\beta}=\left[\begin{array}{cc}
\cos (\beta) & \sin (\beta) \\
-\sin (\beta) & \cos (\beta)
\end{array}\right] .
$$


Then, the expectation in (99) over the uniformly distributed random variable $\beta$ can be expressed as

$$
E_{\beta}\left[\mathbf{G}_{\beta} \mathbf{A G}_{\beta}^{T}\right]=\left[\begin{array}{ll}
b_{11} & b_{12} \\
b_{21} & b_{22}
\end{array}\right]
$$

Now, taking the expectation over the uniform distribution of $\beta$,

$$
\begin{array}{ll}
b_{11}=\frac{a_{11}+a_{22}}{2}, & b_{12}=\frac{a_{12}-a_{21}}{2} \\
b_{21}=\frac{a_{21}-a_{12}}{2}, & b_{22}=\frac{a_{11}+a_{22}}{2} .
\end{array}
$$

For $\mathbf{A}$ a symmetric matrix, we finally obtain

$$
E_{\beta}\left[\mathbf{G}_{\beta} \mathbf{A G}_{\beta}^{T}\right]=\left[\begin{array}{cc}
\frac{a_{11}+a_{22}}{2} & 0 \\
0 & \frac{a_{11}+a_{22}}{2}
\end{array}\right]=\frac{1}{2} \operatorname{tr}\{\mathbf{A}\} \cdot \mathbf{I}
$$

\section{APPENDIX D}

\section{AVERAGING Over DiRECTIVE COSINES}

This Appendix proves that $\overline{\mathbf{C}_{\mathcal{N}}^{-1}}$, defined in (66), presents a closed-form solution as

$$
\overline{\mathbf{C}_{\mathcal{N}}^{-1}}=E_{\boldsymbol{\alpha}}\left[\left(\mathbf{F}^{T} \mathbf{A F}\right)\right]=\frac{1}{2 c^{2}} \operatorname{tr}\{\mathbf{A}\} \cdot \mathbf{I}
$$

We have ? $<>$ dtex $><$ ?CDATA

$$
\begin{aligned}
\mathbf{F}^{T} \mathbf{A} \mathbf{F} & =\sum_{k, k^{\prime}}[\mathbf{A}]_{k, k^{\prime}} \mathbf{f}_{k} \mathbf{f}_{k^{\prime}}^{T} \\
E_{\boldsymbol{\alpha}} \mathbf{F}^{T} \mathbf{A} \mathbf{F} & =\sum_{k, k^{\prime}}[\mathbf{A}]_{k, k^{\prime}} E_{\boldsymbol{\alpha}} \mathbf{f}_{k} \mathbf{f}_{k^{\prime}}^{T}
\end{aligned}
$$

where $[\mathbf{A}]_{k, k^{\prime}}$ is the $k$ th column and $k^{\prime}$ th row element of $\mathbf{A}$, and where $\mathbf{f}_{k}$ is the $k$ th row of $\mathbf{F}$ defined from (51) as

$$
\mathbf{f}_{k}=\frac{1}{c}\left[\begin{array}{l}
\cos \left(\alpha_{k}\right) \\
\sin \left(\alpha_{k}\right)
\end{array}\right] .
$$

So from (102), we need only evaluate $E_{\boldsymbol{\alpha}} \mathbf{f}_{k} \mathbf{f}_{k^{\prime}}^{T}$. If independent uniformly distributed angles are assumed for the BSs

$$
\begin{aligned}
E_{\boldsymbol{\alpha}} \mathbf{F}^{T} \mathbf{A} \mathbf{F} & =\sum_{k \neq k^{\prime}}[\mathbf{A}]_{k, k^{\prime}}\left(E_{\boldsymbol{\alpha}} \mathbf{f}_{k}\right)\left(E_{\boldsymbol{\alpha}} \mathbf{f}_{k^{\prime}}^{T}\right)+\sum_{k}[\mathbf{A}]_{k, k} E_{\boldsymbol{\alpha}} \mathbf{f}_{k} \mathbf{f}_{k}^{T} \\
E_{\boldsymbol{\alpha}} \mathbf{f}_{k} & =\mathbf{0} \\
E_{\boldsymbol{\alpha}} \mathbf{F}^{T} \mathbf{A} \mathbf{F} & =\sum_{k}[\mathbf{A}]_{k, k} E_{\boldsymbol{\alpha}} \mathbf{f}_{k} \mathbf{f}_{k}^{T}
\end{aligned}
$$

so that only the diagonal components of $\mathbf{A}$ are important, on average. Holding that $\alpha_{k}$ are uniformly distributed, the following equality is true:

$$
\begin{aligned}
E_{\boldsymbol{\alpha}} \mathbf{f}_{k} \mathbf{f}_{k}^{T} & =\frac{1}{c^{2}} E_{\alpha_{k}}\left[\begin{array}{cc}
\cos ^{2} \alpha_{k} & \cos \alpha_{k} \cdot \sin \alpha_{k} \\
\cos \alpha_{k} \cdot \sin \alpha_{k} & \sin ^{2} \alpha_{k}
\end{array}\right] \\
& =\frac{1}{2 c^{2}} \mathbf{I}
\end{aligned}
$$

and we obtain the final expression

$$
\overline{\mathbf{C}_{\mathcal{N}}^{-1}}=E_{\boldsymbol{\alpha}} \mathbf{F}^{T} \mathbf{A} \mathbf{F}=\sum_{k} A_{k, k} \frac{1}{2 c^{2}} \mathbf{I}=\frac{1}{2 c^{2}} \operatorname{tr}(\mathbf{A}) \cdot \mathbf{I}
$$

\section{APPENDIX E}

\section{APPROXIMATION OF $\mathbf{R}$ FOR LARGE $N$}

In this Appendix, we show that the random matrix $\tilde{\mathbf{R}}$, the sorted version of $\mathbf{R}$ defined as

$$
\tilde{\mathbf{R}}=\operatorname{diag}\left(\operatorname{sort}\left[\sigma_{1}^{2}, \sigma_{2}^{2}, \ldots, \sigma_{N}^{2}\right]\right)=\operatorname{diag}\left(\left[\tilde{\sigma}_{1}^{2}, \tilde{\sigma}_{2}^{2}, \ldots, \tilde{\sigma}_{N}^{2}\right]\right)
$$

has stochastic convergence to a deterministic matrix, where the elements of its diagonal can be expressed as

$$
\tilde{\sigma}_{n}=F^{-1}\left(\frac{n}{N}\right)
$$

where $F^{-1}(p)$ is the inverse cumulative function of $f_{\sigma}(\sigma)$.

For a given $n$, let us define $\tilde{\sigma}_{n}^{\prime 2}$ such that $\tilde{\sigma}_{n} \leq \tilde{\sigma}_{n}^{\prime} \leq \tilde{\sigma}_{n+1}$. The probability that $\sigma<\tilde{\sigma}_{n}^{\prime}$ is

$$
\operatorname{prob}\left(\sigma<\tilde{\sigma}_{n}^{\prime}\right)=F\left(\tilde{\sigma}_{n}^{\prime}\right)=\int_{-\infty}^{\tilde{\sigma}_{n}^{\prime}} f_{\sigma}(\sigma) d \sigma .
$$

By the law of large numbers, the $n$th position of $\tilde{\sigma}_{n}^{2}$ in $\left[\tilde{\sigma}_{1}^{2}, \tilde{\sigma}_{2}^{2}, \ldots, \tilde{\sigma}_{N}^{2}\right]$ can be expressed as a function of the previously described probability as follows:

$$
F\left(\tilde{\sigma}_{n}^{\prime}\right)=\lim _{N \rightarrow \infty} \frac{n}{N} .
$$

Taking into account that $\lim _{N \rightarrow \infty} \tilde{\sigma}_{n}=\tilde{\sigma}_{n}^{\prime}=\tilde{\sigma}_{n+1}$, we obtain (105) from (106), as we wanted to prove.

\section{APPENDIX F \\ PROOF OF (31)}

We show here that if $\mathbf{H} \mathbf{1}=\mathbf{0}$, it holds that

$$
\mathbf{R}^{-1}-\frac{\mathbf{R}^{-1} \mathbf{1 1}^{T} \mathbf{R}^{-1}}{\mathbf{1}^{T} \mathbf{R}^{-1} \mathbf{1}}=\mathbf{H}^{T}\left(\mathbf{H} \mathbf{R} \mathbf{H}^{T}\right)^{-1} \mathbf{H} .
$$

Proof: If $\boldsymbol{\Xi}$ is a matrix and $\mathbf{G}$ is any Gram matrix, the matrix $\mathbf{G}_{\Xi}^{\perp}$ is a projection matrix

$$
\mathbf{G}_{\Xi}^{\perp}=\mathbf{G}-\mathbf{G} \boldsymbol{\Xi}\left[\boldsymbol{\Xi}^{T} \mathbf{G} \boldsymbol{\Xi}\right]^{-1} \boldsymbol{\Xi}^{T} \mathbf{G}
$$

that fulfills

$$
\mathbf{G} \Xi \boldsymbol{\Xi}=\mathbf{G} \boldsymbol{\Xi}-\mathbf{G} \boldsymbol{\Xi}\left[\boldsymbol{\Xi}^{T} \mathbf{G} \boldsymbol{\Xi}\right]^{-1} \boldsymbol{\Xi}^{T} \mathbf{G} \boldsymbol{\Xi}=\mathbf{0} .
$$

Hence, for $\mathbf{G}=\mathbf{R}$ and $\boldsymbol{\Xi}=\mathbf{H}^{T}$, we have

$$
\mathbf{R}_{\mathbf{H}^{T}}^{\perp}=\mathbf{R}-\mathbf{R} \mathbf{H}^{T}\left[\mathbf{H} \mathbf{R} \mathbf{H}^{T}\right]^{-1} \mathbf{H R}
$$

and $\mathbf{R}_{\mathbf{H}^{T}}^{\perp} \mathbf{H}^{T}=\mathbf{0}$. Conseguently, $\mathbf{R}_{\mathbf{H}^{T}}^{\perp}$ has rank one (because $\mathbf{H}$ is almost full rank) and $\mathbf{R}_{\mathbf{H}^{T}}^{\perp}=\lambda \mathbf{a} \mathbf{a}^{T}$, with $\mathbf{H a}=\mathbf{0}$. Therefore, $\mathbf{a}=\mathbf{1}$ fulfills the required orthogonality with the columns of $\mathbf{H}$ and

$$
\mathbf{R}-\mathbf{R H}^{T}\left[\mathbf{H R H} \mathbf{H}^{T}\right]^{-1} \mathbf{H R}=\lambda \mathbf{1 1}^{T} .
$$

Multiplying by $\mathbf{R}^{-1}$ on both sides

$$
\mathbf{R}^{-1}-\mathbf{H}^{T}\left[\mathbf{H R} \mathbf{H}^{T}\right] \mathbf{H}=\lambda \mathbf{R}^{-1} \mathbf{1 1}^{T} \mathbf{R}^{-1} .
$$

Now, as $\mathbf{H} \mathbf{1}=\mathbf{0}$, multiplication by $\mathbf{1}^{T}$ and $\mathbf{1}$ on both sides yields $\mathbf{1}^{T} \mathbf{R}^{-1} \mathbf{1}=\lambda \mathbf{1}^{T} \mathbf{R}^{-1} \mathbf{1 1}^{T} \mathbf{R}^{-1} \mathbf{1}$. Thus, $\quad \lambda=$ $\left(\mathbf{1}^{T} \mathbf{R}^{-1} \mathbf{1}\right)^{-1}$, and

$$
\mathbf{R}^{-1}-\mathbf{H}^{T}\left[\mathbf{H R H}^{T}\right]^{-1} \mathbf{H}=\frac{\mathbf{R}^{-1} \mathbf{1 1}^{T} \mathbf{R}^{-1}}{\mathbf{1}^{T} \mathbf{R}^{-1} \mathbf{1}} .
$$




\section{APPENDIX G}

\section{DERIVATION OF (67)}

In this Appendix we prove (67)

$$
\overline{\mathbf{C}_{\mathcal{N}}}>\left[\overline{\mathbf{C}_{\mathcal{N}}^{-1}}\right]^{-1}
$$

From the definition of $\overline{\mathbf{C}_{\mathcal{N}}}$ and $\overline{\mathbf{C}_{\mathcal{N}}^{-1}}$ in (54) and (66), respectively, we have

$$
\begin{aligned}
& \overline{\mathbf{C}_{\mathcal{N}}}=E_{\boldsymbol{\alpha}}\left[\left(\mathbf{F}^{T} \mathbf{A F}\right)^{-1}\right]=E_{\mathcal{S} \mathcal{S}} E_{\beta}\left[\left(\mathbf{F}^{T} \mathbf{A} \mathbf{F}\right)^{-1}\right] \\
& \overline{\mathbf{C}_{\mathcal{N}}^{-1}}=E_{\boldsymbol{\alpha}}\left[\left(\mathbf{F}^{T} \mathbf{A F}\right)\right]=E_{\mathcal{S S}} E_{\beta}\left[\left(\mathbf{F}^{T} \mathbf{A F}\right)\right]
\end{aligned}
$$

where applying the result obtained in Appendix C, we have

$$
\begin{aligned}
\overline{\mathbf{C}_{\mathcal{N}}} & =\frac{1}{2} E_{\mathcal{S}} \mathcal{S} \operatorname{tr}\left\{\left(\mathbf{F}_{\mathcal{S} \mathcal{S}}^{T} \mathbf{A} \mathbf{F}_{\mathcal{S S}}\right)^{-1}\right\} \cdot \mathbf{I} \\
\overline{\mathbf{C}_{\mathcal{N}}^{-1}} & =\frac{1}{2} E_{\mathcal{S S}} \operatorname{tr}\left\{\left(\mathbf{F}_{\mathcal{S S}}^{T} \mathbf{A} \mathbf{F}_{\mathcal{S S}}\right)\right\} \cdot \mathbf{I}
\end{aligned}
$$

We may now perform the singular value decomposition (SVD) of $\left(\mathbf{F}_{\mathcal{S}}^{T} \mathbf{A} \mathbf{F}_{\mathcal{S S}}\right)$ so that

$$
\begin{aligned}
\left(\mathbf{F}_{\mathcal{S S}}^{T} \mathbf{A} \mathbf{F}_{\mathcal{S S}}\right) & =\mathbf{U}_{\mathcal{S S}} \boldsymbol{\Delta}_{\mathcal{S S}} \mathbf{U}_{\mathcal{S S}}^{H} \\
{\left[\left(\mathbf{F}_{\mathcal{S S}}^{T} \mathbf{A} \mathbf{F}_{\mathcal{S S}}\right)\right]^{-1} } & =\mathbf{U}_{\mathcal{S S}} \boldsymbol{\Delta}_{\mathcal{S S}}^{-1} \mathbf{U}_{\mathcal{S S}}^{H}
\end{aligned}
$$

where, if $\boldsymbol{\Delta}_{\mathcal{S S}}=\operatorname{diag}\left(\mu_{1}, \ldots, \mu_{P}\right)$, we have

$$
\begin{aligned}
& \overline{\mathbf{C}_{\mathcal{N}}}=E_{\mathcal{S S}} \frac{1}{2} \sum_{p=1}^{P} \mu_{p}^{-1} \cdot \mathbf{I} \\
& \overline{\mathbf{C}_{\mathcal{N}}^{-1}}=E_{\mathcal{S S}} \frac{1}{2} \sum_{p=1}^{P} \mu_{p} \cdot \mathbf{I} .
\end{aligned}
$$

The harmonic-arithmetic mean inequality establishes that

$$
\frac{1}{P} \sum_{p=1}^{P} \mu_{p}^{-1}>\left[\frac{1}{P} \sum_{p=1}^{P} \mu_{p}\right]^{-1} .
$$

so for the 2-D case, this is $P=2$, using $E_{S S}\{x\} \geq 1 / E_{S S}\{x\}$, we have

$$
\overline{\mathbf{C}_{\mathcal{N}}}>\left[\overline{\mathbf{C}_{\mathcal{N}}^{-1}}\right]^{-1}
$$

\section{REFERENCES}

[1] D. Torrieri, "Statistical theory of passive location sytems," IEEE Trans. Aerosp. Electron. Syst., vol. AES-20, Mar. 1984.

[2] J. Smith and J. Abel, "Closed-form least-squares source location estimation from range-difference measurements," IEEE Trans. Acoust., Speech, Signal Process., vol. ASSP-35, no. 12, pp. 1661-1669, Dec. 1987.

[3] T. S. Rappaport, J. H. Reed, and B. D. Woerner, "Position location using wireless communications on highways of the future," Commun. Mag., vol. 34, no. 10, pp. 33-41, Oct. 1996.

[4] A. Urruela and J. Riba, "Novel closed-form ML position estimator for hyperbolic location," in Proc. IEEE Int. Conf. Acoustics, Speech, and Signal Processing (ICASSP), vol. 2, Montreal, QC, Canada, 2004, pp. 14952.

[5] K. Chan and Y. T. Ho, "A simple and efficient estimator for hyperbolic location," IEEE Trans. Signal Process., vol. 42, no. 8, pp. 1905-1915, Aug. 1994.
[6] A. Abrardo, G. Benelli, C. Maraffon, and A. Toccafondi, "Performance of TDOA-based radiolocation techniques in CDMA urban environments," in Proc. IEEE Int. Conf. Communications, vol. 1, Rhodes, Greece, 2002, pp. 431-435.

[7] D.-H. Shin and T.-K. Sung, "Comparisons of error characteristics between TOA and TDOA positioning," IEEE Trans. Aerosp.Electron. Syst., vol. 38 , no. 1, pp. 307-311, Jan. 2002.

[8] M. A. Spirito, "On the accuracy of cellular mobile station location," IEEE Tran. Veh. Technol., vol. 50, no. 3, pp. 674-685, May 2001.

[9] M. Deffenbaugh, J. G. Bellingham, and H. Schmidt, "The relationship between spherical and hyperbolic positioning," in Proc. OCEANS'96. MTS/IEEE. Prospects for the 21st Century, vol. 2, 1996, pp. 590595.

[10] T. Silventoinen and M. I. Rantalainen, "Mobile station emergency locating in GSM," in Proc. IEEE Int. Conf. Personal Wireless Communications, New Delhi, India, 1996, pp. 232-238.

[11] L. Lopes, E. Viller, and B. Ludden, "GSM standards activity on location," in Proc. Inst. Elect. Eng. (IEE) Novel Methods of Location and Tracking of Cellular Mobiles and Their System Applications, 1999, pp. 7/1-7/7.

[12] G. Caffery and J. J. Stuber, "Overview of radiolocation in CDMA cellular systems," IEEE Commun. Mag., vol. 36, no. 4, pp. 38-45, Apr. 1998.

[13] Y. Zhao, "Standardization of mobile phone positioning for 3G systems," IEEE Commun. Mag., vol. 40, no. 7, pp. 108-116, Jul. 2002.

[14] C. Botteron, M. Fattouche, and A. H. Madsen, "Statistical theory of the effects of radio location system design parameters on the positioning performance," in Proc. Vehicular Technology Conf., vol. 2, Vancouver, BC, Canada, 2002, pp. 1187-1191.

[15] H. Koorapaty, H. Grubeck, and M. Cedervall, "Effect of biased measurement errors on accuracy of position location methods," in Proc. Global Telecommunications Conf., vol. 3, Sydney, Australia, 1998, pp. 14971502.

[16] L. J. Greenstein, V. Erceg, Y. S. Yeh, and M. V. Clark, "A new pathgain/delay-spread propagation model for digital cellular channels," IEEE Trans. Veh. Technol., vol. 46, no. 2, pp. 477-485, May 1997.

[17] L. L. Scharf, Statistical Signal Processing. Detection, Estimation and Time Series Analysis. Reading, MA: Addison-Wesley, 1991.

[18] J. R. Magnus and H. Neudecker, Matrix Differential Calculus, Wiley Series in Probability and Statistics. New York: Wiley.

[19] J. J. Caffery Jr., "A new approach to the geometry of TOA location," Proc IEEE Vehicular Technology Conf., Boston, MA, vol. 4, 2000.

[20] M. Aso, M. Kawabata, and T. Hattori, "A new location estimation method based on maximum likelihood function in cellular systems," in Proc. IEEE Vehicular Technology Conf., vol. 1, VTC 2001 Fall, IEEE VTS 54th, 2000, pp. $106-110$

[21] L. Cong and W. Zhuang, "Hybrid TDOA/AOA mobile user location for wideband CDMA cellular systems," IEEE Trans. Wireless Commun., vol. 1, no. 3, pp. 439-447, Jul. 2002.

[22] K. W. Cheung, H. C. So, W. K. Ma, and Y. T. Chan, "Least squares algorithms for time-of-arrival-based mobile location," IEEE Trans. Signal Process., vol. 52, no. 4, pp. 1121-1130, Apr. 2004.

[23] B. T. Fang, "Simple solutions for hyperbolic and related position fixes," IEEE Trans. Aerosp. Electron. Syst., vol. 26, no. 5, pp. 748-753, Sep. 1990.

[24] J. Abel and J. Smith, "The spherical interpolation method for closedform passive source localization using range difference measurements," in Proc. IEEE Int. Conf. Acoustics, Speech, and Signal Processing (ICASSP), vol. 12, Dallas, TX, Apr. 1987, pp. 471-474.

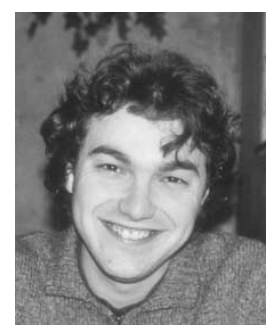

Andreu Urruela (S'01) was born in Castellbisbal, Barcelona, Spain, in 1978. He received the M.Sc. degree in telecommunications engineering in 2001 from the Technical University of Catalonia (UPC) Barcelona. He is currently working toward the Ph.D. degree at UPC.

Since September 2001, he has been a Graduate Research Assistant in the Signal Processing for Communications Group at UPC under a Spanish Government predoctoral scholarship. He has been involved in the IST European Mobile Integrated Location System (EMILY) project for the development of advanced algorithms for wireless location as a member of the Signal Processing Group at UPC. His research interests include high accuracy time delay estimators, closed-form algorithms for wireless location, and the development of wireless location schemes for cellular-networks robust to multipath and NLOS. 


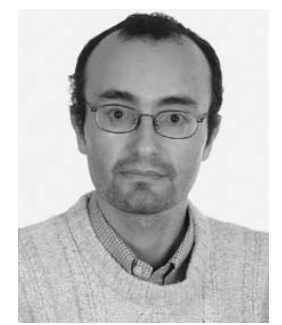

Josep Sala (M'86) was born in Callús, Spain, in 1967. $\mathrm{He}$ received the M.Sc. and Ph.D. degrees in telecommunications engineering from the Technical University of Catalonia (UPC), Barcelona, Spain, in 1991 and 1995, respectively.

During 1992, he was worked at the European Space Operations Centre (ESOC) of the European Space Agency (ESA), Darmstadt, Germany, in the area of software engineering for telemetry processing. From 1993 to late 1994, he held a grant from the Generalitat de Catalunya in support of the Ph.D. degree at the Department of Signal Theory and Communications, UPC. In 1994, he joined this department as Assistant Professor and was promoted to Associate Professor in 1997. He has participated in 12 space-related communication projects for ESA and in 12 other wireless communications projects at the national/European level with industry and institutions. His current research interests are in the field of signal processing, communications, and information theory.

Dr. Sala is recipient of the IEEE Signal Processing Society Best (Senior) Paper Award 2003, the International Symposium on Turbo-Codes and Applications (ISTC'03) Best Poster Paper Award, and the Best Ph.D. Thesis in Telecommunications National Award (Spain, 1995).

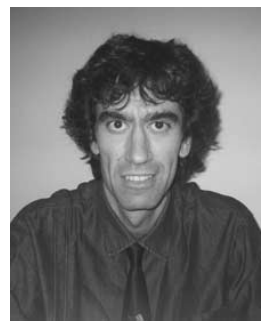

Jaume Riba (SM'05) was born in Barcelona, Spain, in 1966. He received the M. Sc. and Ph.D. degrees in telecommunications engineering from the Technical University of Catalonia (UPC), Barcelona, Spain, in 1992 and 1997, respectively.

In 1992 he entered the Department of Signal Theory and Communications (TSC) and the Signal Processing and Communications Group (SPC), UPC, as Assistant Professor and was promoted to Associate Professor in 1997. His current research interests are in the area of signal processing, communications, and wireless location, with particular emphasis on array processing and digital synchronization techniques. He has been involved in several signal processing research and development projects with European Space Agency research programs. He was the Principal Investigator of the IST project European Mobile Integrated Location System (EMILY) at UPC (2001-2004).

Dr. Riba was a Guest Editor for a Special Issue for Signal Processing for Positioning and Navigation with Applications to Communications. He is a recipient of the 2003 Best Paper Award from the IEEE Signal Processing Society. He has served as Associate Editor of the IEEE TRANSACTIONS ON Signal PROCESSING since 2003 